Erschienen in: Harras, Gisela (Hrsg.): Die Ordnung der Wörter. Kognitive und lexikalische Strukturen. - Berlin, New York: de Gruyter, 1995. S. 227-270. (Institut für deutsche Sprache. Jahrbuch 1993)

\title{
REINHARD BLUTNER
}

\section{Prototypen und Kognitive Semantik}

\section{Einleitung}

Die Absicht dieses Aufsatzes ist es, Gebrauch und Mißbrauch der sogenannten Prototypidee in der lexikalischen Semantik etwas näher zu beleuchten und die sinnvolle Verwendung der Idee von der irreführenden und unsinnigen zu unterscheiden. Ein Problem bei diesem Unterfangen besteht darin, daB es eigentlich ziemlich unklar ist, worin der Inhalt der Prototypidee besteht. Was ein Prototyp eigentlich ist - darüber scheint es mindestens so viele Auffassungen zu geben wie Autoren. Einige Prototypensemantiker (z.B. Geeraerts 1987) haben aus dieser Not eine Tugend gemacht und davor gewarnt anzunehmen, daß die Begriffe der Prototypentheorie "klassische" Definitionen hätten. Um konsistent zu sein, müßten wir es auch akzeptieren, daß derartige wissenschaftliche Begriffe eine Prototypstruktur besitzen. Dazu ist natürlich zu sagen, daß wir nicht erst seit Tarski wissen, zu welch fatalen Konsequenzen die Vermischung von Objekt- und Metaebene führen kann. Im gegenwärtigen Zusammenhang ist diese Vermischung allerdings leicht zu erkennen und es ist ganz offensichtlich, daß sich Wissenschaft mit einem gewissen Anspruch an Seriosität eben erst dann betreiben läßt, wenn der Versuch gemacht wird, Vagheit und Unbestimmtheit aus der wissenschaftlichen Begriffsbildung - wenigstens bis zu einem gewissen Grad - zu eliminieren und es gelingt, diese Grenzen des 'common sense' zu überwinden.

Wenn eine Idee vage und unbestimmt ist, ist es natürlich schwierig, über die sinnvolle und unsinnige Verwendung der Idee zu urteilen. Um diese Schwierigkeit im Zusammenhang mit der Prototypidee etwas zu vermindern, ist es nützlich, (a) etwas über den Ursprung der Idee zu sagen, (b) mögliche Explikationen der Prototypidee zu beachten, auch wenn sie unzulänglich sind, und (c) die Verwendung der Idee innerhalb der lexikalischen Semantik mit der Verwendung anderer Ideen zu kontrastieren.

\subsection{Zum Ursprung der Prototypidee}

Gegenwärtige lexikalische Semantiker, die sich auf die Prototypidee beziehen, suchen die Ursprünge dieser Idee gewöhnlich in der Philosophie (typischerweise in Wittgensteins Spätwerk) und/oder in der Experimentalpsychologie (z.B. Rosch 1975, 1977, 1978) und vergessen dabei, sich auf bedeutende Vertreter der philologischen Tradition zu besinnen, z.B auf Hermann Paul und Karl Otto Erdmann. 


\begin{abstract}
„Denn welche Theorie über Wesen, Bedeutung und Entstehung der Begriffe man auch vertreten mag: vom Standpunkt der Logik wird man immer fordern müssen, daß sie eine unzweideutige, klare Grenze aufweisen, daß sie einen bestimmten Inhalt und Umfang haben. Und Begriffe dieser Art werden durch Worte nicht ohne weiteres bezeichnet. Worte sind vielmehr im allgemeinen Zeichen für ziemlich unbestimmte Komplexe von Vorstellungen, die in mehr oder minder loser Weise zusammenhängen. ... Die Grenzen der Wortbedeutung sind verwaschen, verschwommen, zerfließend. Treffender aber noch wird meines Erachtens der Sachverhalt gekennzeichnet, wenn man überhaupt nicht von Grenzlinien des Umfangs redet, sondern ... von einem Grenzgebiet, daB einen Kern einschließt. Veranschaulicht man sich gewöhnlich den Umfang eines logisch vollkommenen Begriffs durch eine scharfe Kreislinie, wie sie annähernd ein gutgespitzter Bleistift erzeugt, so kann man sich die Abgrenzung eines Wortumfanges durch einen mehr oder minder breiten, in sich zurücklaufenden Streifens versinnlichen, wie ihn ein in Farbe getauchter Pinsel auf einer Fläche hinterläft. (Erdmann 1901, S. 4-5)
\end{abstract}

Die Verbindung zur Prototypidee, wie sie gegenwärtig im Blickpunkt steht, ist so offensichtlich, daß eine Kommentierung überflüssig ist.

Ich will nun etwas ausführlicher auf die Rolle von Prototypen in der experimentalpsychologischen Forschung eingehen, da die gegenwärtige lexikalische Semantik nicht ohne diesen Hintergrund denkbar ist. Ende der 60er Jahre vollzog sich innerhalb eines wichtigen Teilbereichs der Experimentalpsychologie, der Begriffsbildungsforschung, ein Paradigmenwechsel, in dessen Verlauf das sogenannte klassische Paradigma der Begriffsbildung (z.B. Bruner/Goodnow/Austin 1956) durch das Prototypen-Paradigma (z.B. Rosch 1975, 1977, 1978) verdrängt wurde. Ein Beispiel aus der Spätzeit des klassischen Paradigmas, als die Maschinelle Intelligenz begann, sich mit der experimentellen Begriffsbildungsforschung anzufreunden (vgl. z.B. Hunt 1962; Shepart u.a. 1961), soll ausreichen, um den Ausgangspunkt für die von Rosch eingeleitete $W$ e n $d$ e des Forschungsprogramms zu verdeutlichen. Der obere Teil von Abbildung 1 zeigt geometrische Figuren, die als positive bzw. negative Instanzen für eine (künstliche) Kategorie $K$ aufzufassen sind. In einem typischen Begriffsbildungsexperiment werden Versuchspersonen in zufälliger Reihenfolge mit diesen Figuren konfrontiert. Ihre Aufgabe besteht darin zu entscheiden, ob die einzelnen Figuren zur Kategorie $K$ gehören oder nicht. Der Versuchsleiter gibt eine entsprechende Rückmeldung, die sich an der vorgegebenen Struktur von $K$ orientiert. Nach einiger Zeit stabilisiert sich das Antwortverhalten der Versuchspersonen, sie haben die Kategorie $K$ e r w o r b e $n$. Die interessante psychologische Frage ist, worin das im Experiment erworbene Wissen besteht. Mit Hilfe unterschiedlicher Techniken (Transfer, Reaktionszeit) wurde die These gestützt, daß 
die Versuchspersonen dabei gewisse Merkmale extrahieren (im Beispiel sind dies Merkmale über Form und Größe - Farbe wird als irrelevant erkannt) und mit diesen Merkmalen Regeln für die $\mathrm{r}$ i c h t i g e Klassenzuordnung bilden. Im unteren Teil von Abbildung 1 ist eine derartige Entscheidungsstruktur (sie entspricht einer logischen Disjunktion) dargestellt. Auf welche Weise die relevanten Merkmale extrahiert werden und wie der schrittweise Aufbau der zugrunde gelegten Entscheidungsstrukturen geschieht - das sind zentrale Fragen, die das klassische Paradigma der Begriffsbildung gestellt und zu beantworten versucht hat.
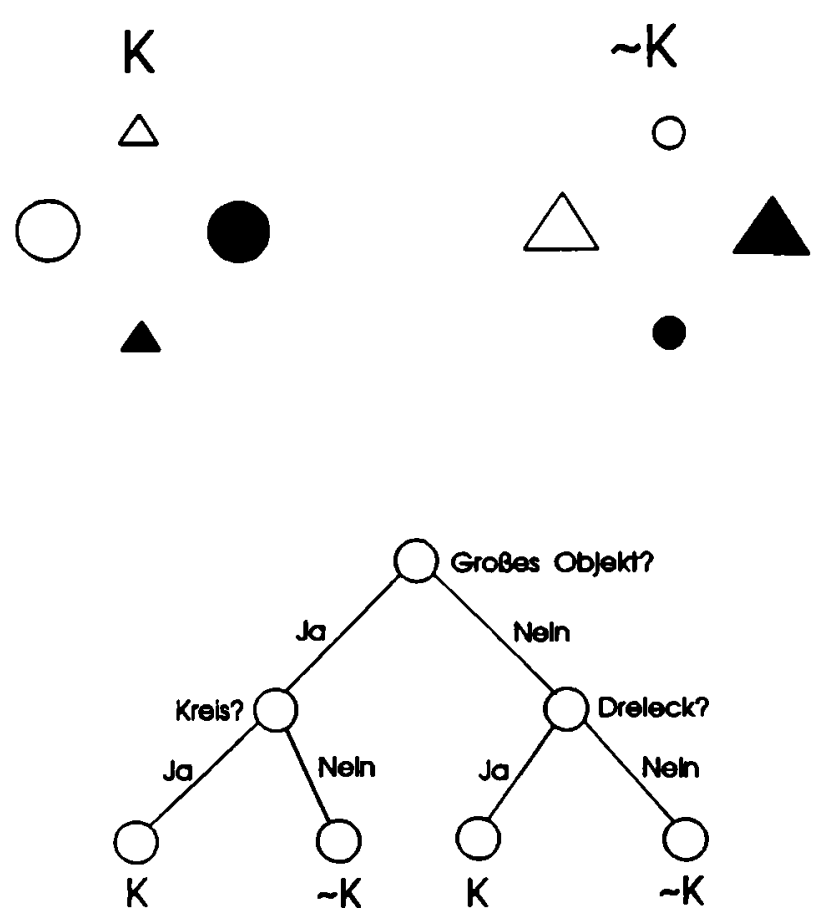

K: (GROB \& KREIS) v (KREIS \& DREIECK)

Abbildung 1: Illustration zum klassischen Paradigma der Begriffsbildung 
Im Prinzip lassen sich beliebige Zerlegungen einer gegebenen Domäne D von Objekten in distinkte Kategorien X, Y, ... durch Entscheidungsstrukturen im Sinne des klassischen Paradigmas ausdrücken. Die Frage ist, ob diese Strukturen tatsächlich immer als plausible Kandidaten für interiorisierte Kenntnisstrukturen taugen (psychologische Realität) und ob die ausschließlich zu Experimentierzwecken konstruierten Kategorien trotz ihres künstlichen Charakters geeignet sind, etwas über die interne Strukturbildung bei der wirklichen Auseinandersetzung mit unserer alltäglichen Umwelt auszusagen (ökologische Validität). Die Überzeugung, daß diese Frage in beiden Teilen mit Nein zu beantworten ist, löste die Krise des klassischen Paradigmas der Begriffsbildung aus. Im Zusammenhang mit der Beantwortung des ersten Teils dieser Frage mag folgendes fiktive Experiment zur Verdeutlichung dienen. Man denke sich eine Kartegorie $\mathrm{X}$ und eine entsprechende Kontrastkategorie $\mathrm{Y}$ wie sie in Abbildung 2 gegeben sind.

Es ist nun ziemlich unplausibel anzunehmen, daß nach einer entsprechenden Lernprozedur eine Entscheidungsstruktur der Art ausgebildet wird, wie sie das klassische Paradigma vorschreibt. Der untere Teil von Abbildung 2 stellt einen entsprechenden (minimalen) Entscheidungsbaum dar. Er beschreibt die logische Bedingungen für die Zugehörigkeit zu X bzw. Y jeweils in Form einer dreifachen Disjunktionen. Viel plausibler als die Annahme derartig komplexer klassischer Strukturen sind Mechanismen, welche die Kategorienbildung auf die $\mathrm{t} y \mathrm{p}$ i $\mathrm{s} \mathrm{ch}$ e $\mathrm{n}$ oder c harakteristis chen Merkmale der Kategorien $\mathrm{X}$ bzw. $\mathrm{Y}$ zurückführen. So ist die Kategorie $\mathrm{X}$ wohl am besten durch die typischen Merkmale große Ohren, schielt, rundes Gesicht etc. gegeben und die Kategorie Y durch die typischen Merkmale kleine Ohren, schielt nicht, ovales Gesicht etc. Im Beispiel werden durch diese Merkmale die ins Zentrum der Kategorien X und Y gesetzten Objekte beschrieben, die als Prototypen der betrachteten Kategorien anzusehen sind. Ein zu klassifizierendes Objekt wird derjenigen Kategorie zugeordnet, deren Prototyp es am meisten ähnelt. Dieses Verfahren liefert in unserem Beispiel eine eindeutige Objektklassifizierung, allerdings auf völlig andere Weise als das klassische Verfahren. Darüber hinaus führt die Graduiertheit der Ähnlichkeiten auf einen Gradienten hinsichtlich der Typikalität - ein Phänomen, das sich in Abbildung 2 (oberer Teil) andeutet und für das es im klassischen Paradigma keine Erklärung gibt. Die eben skizzierten Gedanken zur Kategorisierung mit Hilfe von Prototypen sind durch zahlreiche psychologische Experimente bestätigt worden (z.B. mit schematisierten Gesichtern, zweidimensionalen Punktmustern, Buchstabenketten; für eine Übersicht s. Smith/Medin 1981). 


\section{$X$}
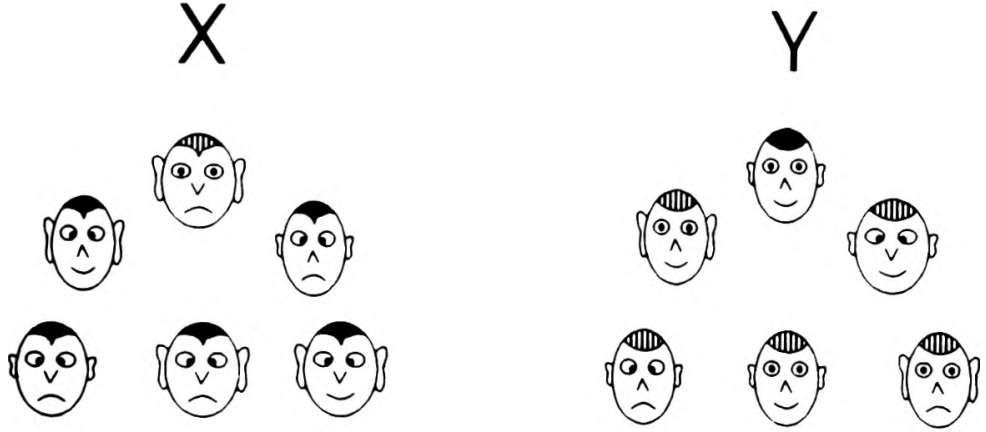

$\left\{\begin{array}{c}0 \\ v\end{array}\right)$
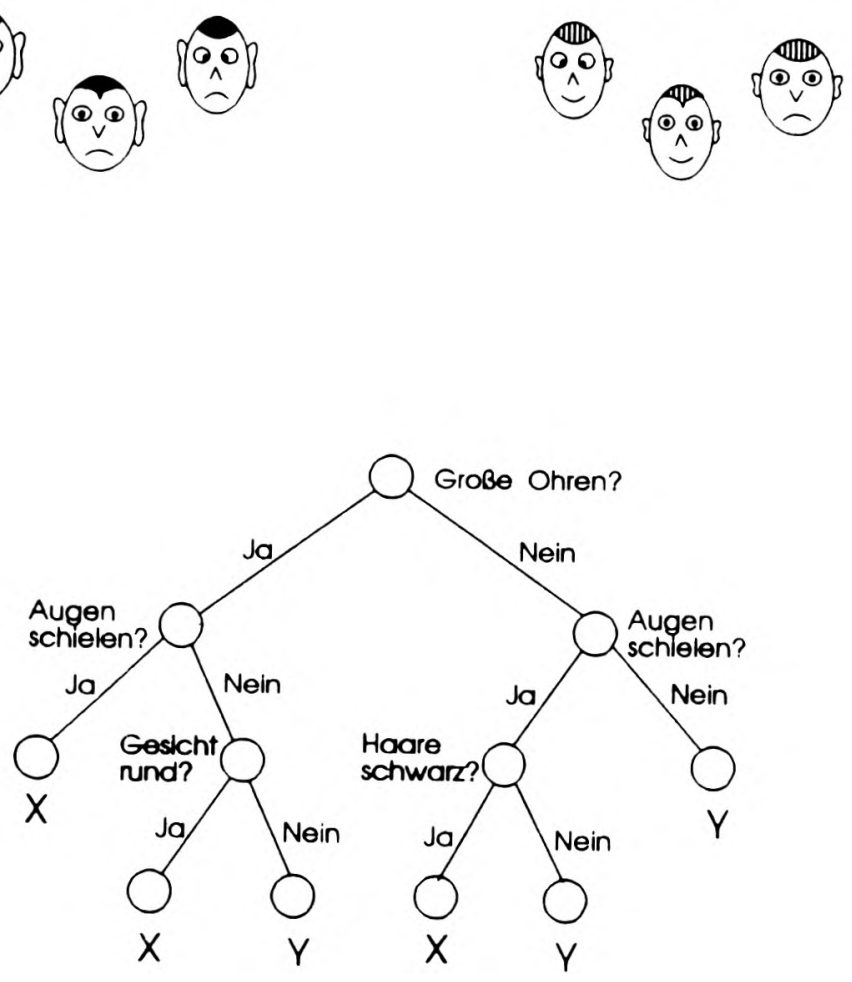

Abbildung 2: Durch Prototypen organisierte Kategorien und klassische Entscheidungsstruktur 
Die Untersuchung natürlicher Taxonomien - Abbildung 3 zeigt einfache Beispiele - belegt die ö $\mathrm{kol}$ og is che Validität der Rolle von Prototypen bei der Kategorienbildung.

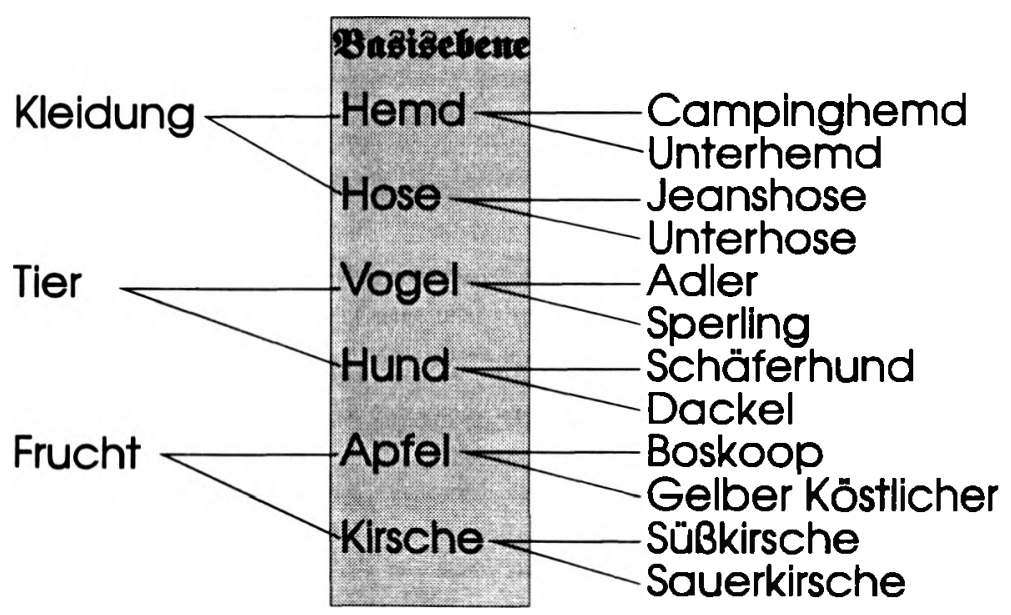

Abbildung 3: Natürliche Taxonomien

Die Arbeiten von Rosch und anderen (z.B. Rosch 1978; Rosch/Mervis 1975, Roseh/Mervis/Gray/Johnson/Boyes-Bream 1976) haben zwei Dimensionen der strukturellen Organisation von natürlichen Taxonomien demonstriert, die horizontale und die vertikale Dimension. Die horizontale Dimension betrifft die Zerlegung einer konzeptuellen Domäne in Kategorien einer bestimmten Abstraktionsstufe (z.B. die Zerlegung der Klasse der Kleidungsstücke in die Kategorien Hemd, Hose, Strümpfe usw.). Prototypen spielen eine Schlüsselrolle bei der horizontalen Organisation. Die Kategorien einer bestimmten Abstraktionsstufe tendieren nämlich dazu, durch prototypische Instanzen definiert zu werden, welche die Redundanzstruktur der Kategorien gewissermaßen als Ganzes 
widerspiegeln. Die vertikale Dimension eines Kategoriensystems beinhaltet die unterschiedliche Rolle verschiedener Abstraktionsebenen (Kategorisierungsebenen). Innerhalb eines hierarchischen Kategorisierungssystems gibt es eine sogenannte Basisebene der Kategorisierung, die sich durch besondere Eigenschaften auszeichnet. Die Kategorien der $\mathrm{B}$ a $\mathrm{s}$ i s e b e $\mathrm{n}$ e besitzen ein hohes $\mathrm{MaB}$ an innerer Familienähnlichkeit und heben sich dennoch gut von entsprechenden Kontrastkategorien ab. Kategorien der Basisebene sind funktionell und epistemologisch primär: Sie sind die allgemeinsten Kategorien, für die konsistente mentale Bilder und konsistente Motorprogramme existieren. Gemessen an der Kategorisierung Erwachsener sind sie die ersten stabilen Kategorien, die Kinder beherrschen und für die Namen ausgebildet werden.

\subsection{Der "Paradigmenwechsel” innerhalb der lexikalischen Semantik}

Der eben skizzierte Paradigmenwechsel hat die Methoden zur Untersuchung und die Modelle zur Beschreibung begrifflicher Kategorisierungen innerhalb der experimentellen Psychologie grundlegend revolutioniert. Welche Konsequenzen ergeben sich daraus für die linguistische Semantikforschung, insbesondere für die lexikalische Semantik? Glaubt man den Vertretern der Kognitiven Semantik (z.B. Fillmore 1975; Lakoff 1987; Langacker 1986), dann erzwingen die skizzierten Einsichten der experimentellen Psychologie einen radikalen Wandel innerhalb des sprachwissenschaftlichen Denkens, in dessen Verlauf beinahe alle Grundsätze und Methoden der sogenannten Standardauffassung der Semantik (z.B. Katz/Fodor 1963; Katz 1972; Montague 1974) über Bord zu werfen und durch neue zu ersetzen seien.

Zweifellos bedarf die lexikalische Semantik neuer Leitmotive, da die Standardauffassung typischerweise von Idealisierungen, Abstraktionen und Fiktionen Gebrauch macht, die faktisch nicht zutreffen. Ob damit jedoch alle Grundprinzipien der Standardauffassung, insbesondere jene der modelltheoretischen Semantik passé sind, ist eine ganz andere Frage, die sorgfältig untersucht werden muß (s. Abschnitt 2). An dieser Stelle sollen zunächst nur zwei vorläufige Thesen formuliert werden, die gewissermaßen das neue Leitmotiv anklingen lassen, das in verschiedenen Variationen innerhalb der Kognitiven Semantik ertönt.

These 1

Die Bildung sprachlicher Einheiten und Kategorien beruht auf der Interaktion sehr genereller, tendenziell nicht-sprachspezifischer ( $d$.h. allgemein kognitiver) Prinzipien und Mechanismen. 


\section{These 2}

Eines dieser allgemein kognitiven Prinzipien ist mit der Protot y $p$ i d e e; verbunden:

Eine Kategorie ist durch ein bestimmtes Muster (Prototyp) bestimmt, das die Struktur der Kategorie quasi als Ganzes reflektiert. Elemente einer gegebenen Domäne werden der Kategorie aufgrund von Ähnlichkeitsbeziehungen zugeordnet, die den Grad der Übereinstimmung mit dem Prototypen reflektieren.

Zur Präzisierung der zweiten These, die auf die Formulierung eines allgemein-kognitiven Kategorisierungsprinzips hinausläuft (d.h. eines Domänen-übergreifenden Prinzips, das für phonologische, syntaktische, morphologische, konzeptuelle und perzeptive Kategorien in gleicher Weise gilt), greife ich auf eine Idee von Osherson/Smith (1981) zurück. Die dort vorgeschlagene Explikation der Prototypidee beschreibt eine Kategorie K als Quadrupel $\langle\mathrm{D}, \mathrm{d}, \mathrm{p}, \mathrm{c}\rangle$, wobei D die Domäne der Kategorie K darstellt (das Feld der „zu betrachtenden" Objekte); p - der Prototyp der Kategorie - ist ein Element dieser Domäne ( $p \in D)$; d ist eine Distanzmetrik auf $D, d . h . d(x, y)$ ist eine positive reelle Zahl, die das AusmaB der Unähnlichkeit zwischen den Elementen $x$ und $y$ charakterisiert (die Funktion d unterliegt dabei "geometrischen" Restriktionen wie Symmetriebedingung und Dreiecksungleichung); $\mathrm{c}$ ist eine Funktion, die jedem Element von $D$ eine reelle Zahl zwischen 0 und 1 zuordnet, den Grad der Kategorienzugehörigkeit des Elements. Diese Gradangabe muß eine monoton fallende Funktion der Distanz zu p sein, $d . h$., für alle $x$ und $y$ aus $D$ mu $B$ gelten:

$$
\mathrm{d}(\mathrm{x}, \mathrm{p}) \leq \mathrm{d}(\mathrm{y}, \mathrm{p}) \rightarrow \mathrm{c}(\mathrm{y}) \leq \mathrm{c}(\mathrm{x}) .
$$

Ich habe diese Explikation der Prototypidee angeführt, weil sie einfach und instruktiv ist und nicht, weil ich glaube, $\mathrm{da} B$ sie inhaltlich angemessen ist. Manchmal ist es besser, klare Gedanken vor Augen zu haben, auch wenn sie falsch sind, als richtige, die verschwommen und unklar sind.

\subsection{Trugbilder und Herausforderungen}

In den folgenden Abschnitten wird es um Trugbilder und Herausforderungen gehen, die im Zusammenhang mit der Prototypidee stehen. Dabei soll es nicht so sehr um die Unzulänglichkeiten einzelner Explikationen dieser Idee gehen. Daß sich zahlreiche landläufige Vorstellungen über Prototypen als Trugbilder erweisen, darauf wurde verschiedentlich hingewiesen (vgl. z.B. Blutner 1985). Im Mittelpunkt soll vielmehr die generelle Überschätzung der mit dieser Idee verbundenen Erklärungs- 
kraft stehen - eine geeignete Explikation der Idee vorausgesetzt. In diesem Sinne meine ich, daß der von Vertretern der Kognitiven Semantik verkündete "Paradigmenwechsel" innerhalb der lexikalischen Semantik trügerische Hoffnungen weckt, die bei näherem Hinsehen nicht eingelöst werden. In einzelnen Bereichen leistet die Standardauffassung nämlich durchaus befriedigende Analysen, welche die von der Prototypidee durchdrungenen Kognitive Semantik nicht zu leisten vermag. In anderen Bereichen werden die Grenzen b e i d e r Theorien überschritten. Nicht alles, was klassisch unlösbar ist, erfährt durch die Prototypidee eine Lösung.

Um es vorweg zu nehmen, der verkündete Paradigmenwechsel Standardauffassung $\Rightarrow$ Kognitive Semantik scheint mir weniger eine wissenschaftliche Revolution zu sein und eher die Rückkehr zu einem "vorwissenschaftlichen" Stadium der Phänomenbeschreibung. Dennoch kann das mit der Prototypenidee verbundene Kategorisierungsprinzip einen wichtigen Platz innerhalb der lexikalischen Semantik beanspruchen. Damit sind theoretische Herausforderungen verbundenen, die sich zum einen auf eine geeignete Explikation dieses Prinzips beziehen und zum anderen auf die Einordnung des Prinzips in einen theoretischen Rahmen, in dem es wirkt und mit dessen Hilfe es interessante Vorhersagen und Erklärungen liefert. Ich glaube allerdings, daB sich dieser Rahmen eher durch Erweiterung und Modifikation der Standardauffassung ergibt als durch einen radikalen Wandel, der alle bisherigen Grundsätze, besonders die der modelltheoretischen Semantik, über Bord wirft, wie es unter kognitivistischer Flagge neuerdings verlangt wird.

Der Plan für die weiteren Erörterungen ist folgender. Im nächsten Abschnitt werden die Positionen der Standardauffassung mit denen der Kognitiven Semantik verglichen. Dabei werden die Phänomenbereiche benannt, in denen ein Festhalten an traditionellen Positionen geboten ist und diejenigen, wo eine geeignete Explikation der Prototypidee interessante theoretische Innovationen verspricht. Diese Erörterungen legen eine modifizierte Version der Standardauffassung nahe, deren Architektur skizziert wird. In Abschnitt 3 werden exemplarisch Herausforderungen beschrieben, denen sich ein eher konservatives Programm zu stellen hat. Dabei soll zumindest für einige Phänomenbereiche deutlich werden, daB ein modelltheoretischer Ansatz besser in der Lage ist, PrototypPhänomene zu beschreiben und zu erklären als der vorläufig ziemlich diffuse Ansatz der Kognitiven Semantik es vermag. 


\section{Prototypen in der lexikalischen Semantik}

Der nun folgende Vergleich zwischen Standardauffassung und Kognitiver Semantik soll in erster Linie dazu dienen, Platz und Funktion der Prototypidee innerhalb der lexikalischen Semantik näher zu bestimmen und die sinnvolle Verwendung der Idee von der irreführenden zu unterscheiden.

\subsection{Standardauffassung und Kognitive Semantik}

Die Standardauffassung unterscheidet - ich vereinfache und unifiziere - Wörter (im Sinne von Lautformen) von den mit ihnen verbundenen begrifflichen Inhalten (Intensionen) und diese wiederum von Begriffsumfängen (Extensionen), d.h., die mit den einzelnen Begriffsinhalte assoziierten realen oder fiktiven Objekte/Situationen/Ereignisse. Für die interne Strukturierung von Wörtern, Intensionen und Extensionen gelten verschiedene Grundsätze, ebenso für ihre wechselseitigen Beziehungen. Die folgenden Grundsätze sind für die gegenwärtige Diskussion von besonderem Interesse:

- Wörter (Lautformen) sind per Konvention mit bestimmten Intensionen verbunden.

- Intensionen sind e m p i r i s c h mit entspechenden Extensionen assoziiert. Um beispielsweise festzustellen, ob die mit Fritz Müller bezeichnete Person unter die Extension von JUNGGESELLE fält, sind e m p i $\mathrm{is} \mathrm{che} \mathrm{Informationen} \mathrm{notwendig,} \mathrm{die} \mathrm{es} \mathrm{gestatten,}$ zu entscheiden, ob Fritz Müller ein verheirateter Mann ist oder nicht.

- Es lassen sich zwei autonome Kenntnissysteme unterscheiden: (mentales) Wörterbuch und (mentale) Enzyklopädie:

Wörterbuch: Wort (Lautform) $\Rightarrow$ Intension

Enzyklopädie:

Intension $\Rightarrow$ Extension

- Intensionen sind durch Komplexe semantischer Merkmale gegeben. Beispielsweise ist die Intension von Junggeselle durch die semantischen Merkmale MÄNNLICH, ERWACHSEN, UNVERHEIRATET bestimmt. Derartige Merkmalskomplexe haben eine doppelte Funktion: Zum einen stiften sie Sinnrelationen; zum anderen dienen sie als Checklisten für die Bestimmung der Extensionen.

- Extensionen sind $M$ e $n \mathrm{~g}$ e $\mathrm{n}$ von realen/projizierten Entitäten (Objekte, Situationen, Ereignisse). Jeder Begriffsumfang besitzt also eine klare Umgrenzung. Innerhalb des Begriffsumfangs gibt es keine Zentralitätsgradienten, also keine Unterscheidungsmöglichkeiten zwischen den Kernelementen und den eher periphären Elementen der Kategorie. 
Auch die Kognitive Semantik - ich unterstelle, es handelt sich dabei um ein halbwegs einheitliches Theoriengebilde - unterscheidet zwischen der Lautform von Wörtern, damit verbundenen begrifflichen Inhalten und entsprechenden Referenzbereichen. (Ich verwende weiterhin die Ausdrücke I $\mathrm{n} t \mathrm{e} n \mathrm{si}$ i n und $\mathrm{E} \times \mathrm{t}$ e $\mathrm{n} \mathrm{s}$ i o $\mathrm{n}$, wenn auch in einem etwas anderen und weniger technischen Sinne als dem der modelltheoretischen Semantik). Die folgenden Grundsätze geben eine ungefähre Charakterisierung der Kognitiven Semantik oder, besser gesagt, eines (fiktiven) Prototypen der Kognitiven Semantik.

- Lexikalische Begriffe haben verschwommene Grenzen. Die Zugehörigkeit von Elementen zu Kategorien (Extension von Begriffen) wird durch graduierte Funktionen beschrieben (Vagheit lexikalischer Begriffe).

- Lexikalische Begriffe besitzen zentrale Instanzen, die die Kategorie als Ganzes repräsentieren. Innerhalb der Kategoriengrenzen sind gewisse Elemente der Kategorie "schlechtere” (weniger typische) Exemplare der Kategorie als andere (Zentralitätsgradient)

- Semantische Merkmalskomplexe (Intensionen) werden durch Operationen der Prototypenbildung organisiert. Die verschiedenen Merkmale eines Komplexes können in unterschiedlichem Grad hervorgehoben sein.

- Es gibt keine strikte Trennung zwischen Lexikon und Enzyklopädie. Die Grenzen zwischen analytisch und synthetisch, definierend und charakterisierend sind fließend.

- Die verschiedenen Sinne lexikalischer Einheiten bilden Cluster sich überlappender Begriffe. Diese Begriffsfamilien sind nach der Prototypidee organisiert.

- Die Grenzen der Begriffsfamilien sind unbestimmt und offen.

Im folgenden Unterabschnitt werden diese Punkte detaillierter erläutert, auf ihre Stichhaltigkeit hin überprüft und mit entsprechenden Aussagen der Standardtheorie verglichen.

2.2 Einige Gesichtspunkte für den Vergleich Standardtheorie - Kognitive Semantik

\subsubsection{Verhältnis Vagheit - Zentralität}

Vagheit und Zentralität sind Etiketten für zwei Klassen von Phänomenen, die in der Literatur häufig miteinander identifiziert werden. Tabelle 
1 illustriert, daB Vagheit und Zentralität als zwei weitgehend unabhänge Charakterisierungen von lexikalischen Begriffen anzusehen sind.

\begin{tabular}{lll}
\hline Wort & \multicolumn{2}{l}{ Vag- Zentra- relevante } \\
& heit lität & Untersuchungen \\
\hline
\end{tabular}

Typ 1:

Quadrat, Kreis, Kugel

Typ 2:

rot, grün, ...;

Stuhl, Tasse, ...

$+\quad+\quad \begin{aligned} & \text { Berlin/Kay } 1969 \\ & \text { Labov } 1973\end{aligned}$

Typ 3:

(un)gradzahlig, ebene $-\quad+\quad$ Armstrong et al. 1983 geometrische Figur, Frau töten, sprechen, ...

Pulman 1983

Typ 4:

$\begin{aligned} & \text { Vogel, Löwe, .. } \\ & \text { Frucht, Gemüse, .. }\end{aligned} \quad \pm \quad+\quad$ Rosch 1973; 1978

Tabelle 1: Vagheit und Zentralität

Zwar gibt es keine vagen Begriffe, die nicht auch vom Phänomen der Zentralität durchdrungen sind; doch alle anderen Kombination der "metabegrifflichen Merkmale" +/- Vagheit und +/- Zentralität sind realisiert. Begriffe vom Typ 1 sind durch die Paarung [-Vagheit, - Zentralität] gekennzeichnet. Sie sind die einzigen Begriffe, die sich mit Hilfe der Standardauffassung beschreiben lassen. Begriffe vom Typ 2 sind durch die Kombination [+Vagheit, +Zentralität] charakterisiert. Sie bilden die "guten" Begriffe im Sinne der Kognitiven Semantik. Das augenfälligste Beispiel für diesen Typ von Begriffen ist mit dem System der Farbwörter verbunden. Hier dienen offenbar fokale Farbwerte in einem mehrdimensionalen „kognitiven Raum” (Miller/Johnson-Laird 1976) als Verankerungspunkte (Prototypen) der entsprechenden Begriffe und die Kategorisierung von Farbeindrücken (Perzepten) ergibt sich aus der Verrechnung von Distanzen hinsichtlich der verfügbaren Fokalpunkte. Vagheit und Zentralität sind dabei zwei Phänomene, die untrennbar mit diesem Verrechnungsmechanismus verbunden sind. Eine andere Klasse von Begriffen vom Typ 2 ist durch die Kategorisierung von Artefakten gegeben (z.B. Labov 1973). Die entsprechenden Prototypen lassen sich wohl am besten als 3D-Modelle im Sinne von Marr (1982) auffassen (vgl. dazu die Ausführungen in Jackendoff 1987, S. 174ff.). 
Interessant sind die Begriffe vom Typ 3. Darunter fallen mathematische Begriffe wie geradzahlig, ungeradzahlig, Primzahl und nominale Artbegriffe wie Mann und Frau. Bei Begriffen diese Typs, besonders bei den mathematischen, würde man zunächst keine reliablen Prototypeffekte erwarten und es käme einem ziemlich unsinnig vor, beispielsweise nach typischen oder untypischen Primzahlen zu fragen. Eine Studie von Armstrong/Gleitman/Gleitman (1983) hat an dieser Stelle den "gesunden Menschenverstand" korrigiert und gezeigt, daB bei diesen Begriffen mit wohldefinierten Grenzen innerhalb der Begriffsgrenzen durchaus deutliche Typikalitätsunterschiede bestehen können.

\begin{tabular}{|c|c|c|c|}
\hline \multicolumn{2}{|l|}{ Obst } & \multicolumn{2}{|c|}{ gerade Zahl } \\
\hline Apfel & 6.7 & zwei & 7.0 \\
\hline Erdbeere & 5.8 & vier & 6.9 \\
\hline Pflaume & 5.5 & sechs & 6.3 \\
\hline Ananas & 5.3 & tausend & 5.2 \\
\hline Feige & 2.8 & vierunds & 4.9 \\
\hline Olive & 1.6 & $\begin{array}{l}\text { achthun } \\
\text { undsech }\end{array}$ & 4.1 \\
\hline
\end{tabular}

Tabelle 2: Mittlere Typikalitätsschätzungen für Instanzen der Prototyp-Kategorie Obst und Instanzen der "wohldefinierten" Kategorie gerade Zahl. (Nach Daten von Armstrong/Gleitman/Gleitman (1983); beachte, daß dabei englische Termini verwendet wurden.)

Die Autoren haben dazu Roschs (1973) bekanntes Experiment zur Demonstration von Typikalitätseffekten wiederholt, wobei sie sowohl herkömmliche Prototyp-Kategorien verwendeten (Obst, Sport, Pflanze, Auto) als auch im klassischen Sinne "wohldefinierte" Kategorien (gerade Zahl, ungerade Zahl, Frau, ebene geometrische Figur). Die Versuchspersonen sollten von jeweils 6 Exemplaren einschätzen, wie gut das gebotene Exemplar die mit der Kategorie verbundene Idee repräsentiert (auf einer 7-Punkteskala: $7=$ sehr gut, $\ldots, 4=$ mittelmäßig,.., $1=$ sehr schlecht). Das Ergebnis: In b e i d e $n$ Fällen ergeben sich signifikante und reliable Typikalitätsunterschiede (s. Tabelle 2).

Die experimentellen Befunde von Armstrong/Gleitman/Gleitman (1983) haben nachhaltig die Diskussion um die wirkliche "Natur” von Begriffen beeinfluBt und sind mit z.T. sehr umstrittenen Spekulationen verbunden worden. Eine $\mathrm{methodolog}$ is $\mathrm{ch}$ e SchluBfolgerung ist jedoch unumstritten: Aus dem Nachweis von Typikalitätseffekten läBt sich nicht ohne weiteres schluBfolgern, daß die $S$ t r u k t u der un- 
tersuchten Begriffe der Prototypidee folgt, d.h., daß P r o t o t y p e n der Kategorisierung zugrunde liegen. Auch „wohldefinierte” Begriffe zeigen Typikalitätsunterschiede und keiner würde aus diesem Grund den Schluß ziehen, daß beispielsweise die herkömmlichen Definitionen von Primzahl und gerade $Z a h l$ kognitiv irrelevant sind und durch von Prototypen organisierte Familienähnlichkeiten ersetzt werden müssen.

Aus der gleichen methodologischen Einsicht heraus halte ich es für verfehlt, bei Verben wie kill, walk und speak die herkömmlichen Definitionen (Merkmalszerlegungen) aufzugeben und Zuflucht bei einem Prototypansatz zu suchen, nur weil bei der Einschätzung der entsprechenden Unterbegriffe Typikalitätsunterschiede auftreten (s. Tabelle 3 ). Natürlich ist es interessant, in diesen und den vorangegangenen Fällen der Frage nachzugehen, worauf diese Typikalitätsunterschiede denn zurückzuführen sind. Sicherlich spielt dabei eine Vielzahl von Faktoren eine Rolle: Gedächtnisund Verarbeitungsfaktoren ebenso wie strukturelle Bedingungen. So ist denkbar, daß unser Stereotypwissen über die mehr oder weniger verbreiteten Arten des Tötens, Sprechens, Laufens usw. Einfluß auf entsprechende Typikalitätsschätzungen nimmt. Und unsere Intuitionen über repräsentative und weniger repräsentative Primzahlen, gerade oder ungerade Zahlen sind von der Existenz verschiedener Zahlsysteme sicherlich ebenso beeinflußt wie von unterschiedlichen Berechnungsmodellen (vgl. Lakoff 1987, S. 149ft).

\begin{tabular}{lrll}
\hline kill & & speak & \\
\hline murder & 6.9 & recite & 5.4 \\
assassinate & 5.9 & mumble & 4.5 \\
execute & 5.2 & shout & 4.5 \\
massacre & 4.7 & whisper & 4.3 \\
sacrifice & 2.8 & drone & 4.0 \\
commit & 2.7 & stutter & 2.6 \\
suicide & & & \\
\hline
\end{tabular}

Tabelle 3: Mittlere Typikalitätsschätzungen für Unterbegriffe von kill und speak (nach Daten von Pulman 1983)

Während für die Begriffe vom Typ 3 ziemlich klar ist, daß hier die Begrenzung ihres Umfangs durch klassische Kategorienstrukturen geliefert wird, werfen die Begriffe vom Typ 4 neue Fragen auf. Begriffe dieses Typs sind z.B. durch natürliche Artbegriffe vertreten, wie sie Rosch (1983) untersucht hat. Diese Begriffe zeigen deutliche Typikalitätseffekte und besitzen - wie die Begriffe vom Typ 3 - eine klare Umgrenzung ihres Umfangs; für Vagheit ist also so gut wie kein Platz. Um es am Bei- 
spiel Vogel zu illustrieren: Die Kategorienzugehörigkeit ist eine diskrete Funktion - etwas ist entweder ein Vogel oder ist es nicht. (Pinguine sind ebenso eindeutig Vögel wie Fledermäuse keine Vögel sind). Es besteht jedoch ein Unterschied zu den Begriffen vom Typ 3, der deutlich wird, wenn neue, bisher nicht bekannte Instanzen zu bewerten sind. Die Frage, wie z.B. eine gewisse Mutante zu kategorisieren ist, die zwar aussieht wie ein Vogel, sich aber sonst ganz anders verhält, zeigt eine prinzipielle Unbestimmtheit, die von Waismann (1952) als of f e n e T ex t u r beschrieben wurde. In Tabelle 1 sind Begiffe dieses Typs provisorisch durch $[ \pm$ Vagheit, +Zentralität] gekennzeichnet. Welche Rolle Prototypen bei der Bestimmung des Umfangs dieser Art von Begriffen spielen, ist eine interessante Frage, auf die ich im nächsten Abschnitt zurückkomme.

\subsubsection{Prototypidee und Begriffsumfang}

Die bisherige Erörterung hat gezeigt, daß zu unterscheiden ist zwischen Vagheit (Grad der Kategorienzugehörigkeit) und Zentralität (Grad der Repräsentativität oder Typikalität). Je nach der Ausprägung dieser Eigenschaften ist es heuristisch sinnvoll, vier Typen von Begriffen auseinanderzuhalten. Ich will nun auf die Nützlichkeit und auf die Grenzen der Prototypidee zu sprechen kommen, soweit das mit dem Problem im Zusammenhang steht, die Kategorienzugehörigkeit zu bestimmen. Dabei erweist sich die eingeführte heuristische Typisierung als ein zwar grobes, aber recht nützliches Orientierungsraster.

Begriffe vom Typ 1 (hier spielt die Prototypidee offensichtlich überhaupt keine Rolle) lasse ich als marginal beiseite. Für Begriffe vom Typ 2 ist die Prototypidee zweifellos nützlich, aber nicht in der auf Osherson/Smith (1981) zurückgehenden Ausführung, die am Ende von Abschnitt 1.2. angegeben wurde. Diese Explikation vermittelt zwar den Zusammenhang zwischen Typikalität und Vagheit, wie er für Begriffe vom Typ 2 charakteristisch ist. Sie berücksichtigt aber nicht die das sogenannte Feldphänomen. Damit ist die Tatsache gemeint, daß eine Kategorisierungsleistung nicht nur von den Prototypen e i n e r Kategorie bestimmt ist, sondern auch von den Prototypen entsprechender Kontrastkategorien abhängt. Angenähert gilt nämlich, da $\beta$ ein Objekt $x$ eher der Kategorie $K$ als der Kontrastkategorie K' zugeordnet wird, je nachdem, ob die Distanz zwischen $x$ und dem Prototypen von $K$ größer oder kleiner ist als die Distanz $z$ wischen $x$ und dem Prototypen von K'. (Für entsprechende Formalisierungen und Verallgemeinerungen dieser Idee vgl. z.B. Kutschera 1975; Lieb 1980; Lutzeier 1981). 
Für Begriffe vom Typ 3 sind sogenannte duale Modelle vorgeschlagen worden (z.B. Armstrong et al. 1983). Dabei ist die Struktur eines Begriffs durch seinen def i $n$ i $t o r$ is $c h$ en $K$ e r $n$ gegeben. Dieser Kern bestimmt den Begriffsumfang - ganz im Sinne der Standardauffassung. Daneben gibt es ein sogenanntes Berechnungsverfahren, das den Typikalitätsgradienten liefern soll. Daß in dieses Berechnungsverfahren vermutlich eine Reihe ganz unterschiedlicher Mechanismen involviert ist, wurde bereits angedeutet.

Bei den Begriffen vom Typ 4 ist es weitgehend ungeklärt, auf welcher Grundlage die Extension bestimmt ist. Sicher sind hier in der Regel keine Nominaldefinitionen wirksam wie bei den Begriffen vom Typ 3. In vielen Fällen reichen Stereotype (z.B. Putnam 1975) oder idealisierte kognitive Modelle (z.B. Lakoff 1987) aus, um - bei expliziter Angabe einiger Ausnahmen - den Begriffsumfang zu bestimmen. Zur Präzisierung des Stereotypbegriffs (oder alternativ: des Begriffs idealisierter kognitiver Modelle) reichen die Explorationen im Rahmen geometrischer Modelle, wie sie im Zusammenhang mit Begriffen vom Typ 2 durchaus nützlich sind (vgl. z.B. Kutschera 1975; Lieb 1980), mit Sicherheit nicht aus (s. Blutner 1985). Eine treffendere Explikation ergibt sich im Rahmen einer nichtmonotonen Logik von Standardannahmen (s. Abschnitt 3).

Tradierte Vorstellungen über den Inhalt von Begriffen messen „Wesensmerkmalen" (the r e a l e s s e n c e ; vgl. 2.B. Kripke 1972) eine entscheidende Rolle bei. Dabei sind meist Begriffe für natürliche Arten im Visier. In Putnams (1975) Analyse wird diese Art von Wissen und seine Rolle zur Bestimmung von Extensionen eher gering geschätzt und (im Rahmen einer soziologischen Theorie der Arbeitsteilung) aus dem Bereich des Alltagswissens in die Privatsphäre des Expertenwissens delegiert. In einer interessanten Serie von Untersuchungen hat Keil (1987) gezeigt, daß die auf "Wesensmerkmalen” basierenden (Referenz-) Definitionen selbst bei jüngeren Kindern verhaltensrelevant sind, wenn geeignete Bedingungen vorliegen.

In den Experimenten von Keil ging es um die Frage, welche Identitätsbedingungen für Artefakte einerseits und für natürliche Arten andererseits ausschlaggebend sind. Dazu wurden mit bestimmten Objekten gewisse „kosmetische” Operationen ausgeführt (verbal und bildlich). Ein Beispiel aus dem Bereich der Artefakte: Ein Sessel wird so zurechtgezimmert, daß er aussieht wie ein Stuhl. Beispiele aus dem Bereich natürlicher Arten: Ein Pferd wird so bemalt und zurechtgestutzt, bis es aussieht wie ein Zebra; ein Igel wird auf schauerliche Weise in die Erde gesteckt uns so zugerichtet, daß er aussieht wie ein Kaktus. Die Versuchspersonen wurden jeweils nach der Verdeutlichung einer Transformation gefragt, ob 
sich dabei die Identität des entsprechenden Objekts geändert hat, ob also aus dem Sessel ein Stuhl geworden ist, aus dem Pferd ein Zebra, aus dem Igel ein Kaktus.

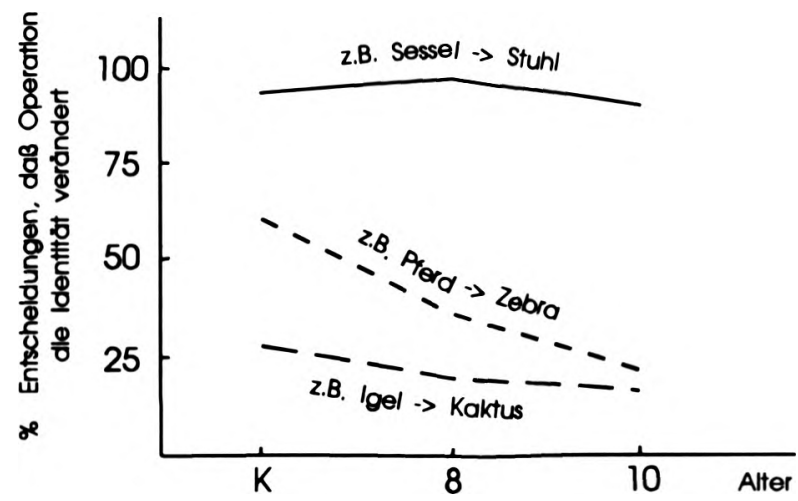

$\begin{array}{ll}\text { Abbildung 4: } & \text { Die Rolle von Wesensmerkmalen und Stereotypwissen bei } \\ & \text { der Beurteilung von Identitätsveränderungen (nach Da- } \\ & \text { ten von Keil 1987) }\end{array}$

Wie nicht anders erwartet, urteilen alle Versuchspersonen, von den Kindergartenkindern bis zu den Erwachsenen, daß sich bei den Artefakten die Identität verändert hat (s. Abbildung 4). Ein ganz anderes Bild entsteht bei den natürlichen Arten. Bei den Transformationen, die sich innerhalb der Gattung Tier (bzw. Pflanze) bewegen, behauptet schon fast die Hälfte der jüngeren Kinder und ein Großteil der älteren Versuchspersonen, daß die ausgeführten Operationen die Identität nicht verändert haben. Bei den Transformationen von Tier-zu Pflanzengestalt (bzw. von Pflanzen- zu Tiergestalt) behauptet selbst die Mehrzahl der Vorschulkinder, daß sich die Identität dabei nicht verändert hat.

Diese Befunde sind aus verschiedenen Gründen interessant. Im gegenwärtigen Zusammenhang ist eine Schlußfolgerung besonders relevant. Die Experimente legen nämlich die Vermutung nahe, daB bei natüriichen Arten die Identitätsbedingungen mit Theorien über das $W$ e s e $n$ d e r A r t zusammenhängen. Obwohl über den Inhalt derartiger 
Theorien bisher nur spekuliert werden kann, demonstrieren die Experimente einleuchtend, dab entsprechende Wesensmerkmale bereits bei Vorschulkindern wirken und das zeigt, daß bereits jüngere Kinder nicht hoffnungslos an Stereotype in Putnams Sinne gebunden sind.

Die bisherige Erörterung hat ihr Ziel erreicht, wenn deutlich geworden ist, daß weder die klassische Vorstellung von Begriffen noch die mit der Prototypidee verbundene Vorstellung für sich genommen ausreichen, um die diskutierten Begriffstypen zu erfassen und ihre Eigentümlichkeiten zu beschreiben. Die Prototypidee als (Vorstufe für ein) Modell der Kategorisierung ist brauchbar für Begriffe vom Typ 2, für Begriffe vom Typ 1 und 3 ist sie überflüssig, für Begriffe vom Typ 4 sind zusätzliche Annahmen erforderlich, nämlich theoretische Annahmen über das Wesen natürlicher Arten. Diese Annahmen liegen in gewisser Weise jenseits von Standardauffassung und Prototypkonzeption. Beachtet man, daB Begriffe vom Typ 2 einen eher kleinen Teil des natürlichen Begriffssystems abdecken, dann wird der Stellenwert der Prototypidee innerhalb einer Theorie der Begriffsstruktur, soweit dies für die lexikalische Semantik relevant ist, nachdrücklich relativiert.

2.2.3 Semantische Merkmalsverknüpfungen: logische Konjunktion oder Operation der Prototypbildung?

Ein wichtiges Problem der lexikalischen Semantik betrifft die Vermittlung zwischen den Lautformen von Wörtern und ihren Referenzbereichen (Extensionen). Entsprechend der Standardauffassung wird diese Vermittlung durch semantische Merkmalskomplexe hergestellt, die per Konvention mit den Lautformen verbunden sind. Diese Merkmalskomplexe sind als Definitionen im klassischen Sinne aufzufassen und dienen bei der Bestimmung der Extensionen als eine Art von "Checklisten". Die semantische Analyse des englischen Worts bachelor hat eine Schlüsselrolle bei der Etablierung dieser Auffassung gespielt (vgl. Katz/Fodor 1963).

Auch die Kognitive Semantik hat sich durch beispielhafte Analysen etabliert. Eine zentrale Rolle spielt dabei die semantische Analyse des Verbs lie/lügen (Coleman/Kay 1981), von der sich mit Recht behaupten läßt, daß sie den Prototypen der P r ot ot y p-S e m a n t ik gestiftet hat. Diese Analyse diente als Muster für unzählige Nachahmungen und fand - vorbehaltlich gewisser Variationen - breite Akzeptanz innerhalb der Kognitiven Semantik.

Ich möchte etwas ausführlicher auf die Studie von Coleman/Kay (1981) eingehen, und zwar aus zweierlei Gründen. Zum einen basiert diese Ana- 
lyse - so meine ich - auf einer Fehleinschätzung des Platzes, den die Prototypidee innerhalb der lexikalischen Semantik einnehmen kann und soll - eine Fehleinschätzung, die typisch für die gesamte Richtung der Kognitiven Semantik ist. Zum anderen scheint mir das gewählte Beispiel besonders geeignet, diesen Irrtum auszuräumen und zu einer differenzierteren Analyse und ansatzweisen Erklärung der beobachteten Phänomene zu gelangen.

Coleman/Kay (1981) entwickeln am Beispiel lie eine semantische Theorie, die Lautformen mit sogenannten „prälinguistischen” kognitiven Schemas (prototype schema, image) assoziiert. Diese Schemas nehmen gewissermaßen die Stelle der semantischen Merkmalskomplexe in der Standardauffassung ein. Wesentlich ist, daß ein derartiges kognitives Schema durch eine Operation der Prototypbildung organisiert wird. Dabei spielen semantische Merkmale durchaus eine Rolle und sie werden durch die formal nicht näher explizierte Operation der Prototypbildung miteinander verknüpft. Dabei können die verschiedenen Merkmale in unterschiedlichem Grade hervorgehoben sein. Um Mißverständnisse hinsichtlich des zentralen Begriffs kognitives Schema zu vermeiden, hier die relevante Passage aus Coleman/Kay (1981):

"The particular prototpye schema which we propose for lie has the following (semi)formal characteristics:

(a) It contains a finite list of properties. In this respect it is like a checklist definition, but in other respects it is not.

(b) The individual properties on the list are each treated as dichotomous, i.e., as either satisfied or not. We envisage, however, that prototype schemata may in general contain gradient properties, whose satisfaction is a matter of degree. We leave open the possibility that an investigation conducted at a more detailed empirical level might find this to be true with lie.

(c) Membership in the category lie is a gradient phenomenon.

(d) Satisfaction of each property on the list contributes to the over-all degree of membership of an individual in the category.

(e) Satisfaction of each property on the list does not necessarily contribute equally to the degree of membership of an individual in the category. That is, properties may be of differential importance in constituting the prototype.

(f) In this gradient framework, the bivalent concept of the 'necessity' and 'sufficiency' of properties do not apply." (Coleman/Kay 1981, S. 27-28).

Im Falle von lügen enthält dieses kognitive Schema die folgenden drei Bestandteile (dabei bezieht sich $S$ auf den Sprecher, A auf den Adressa- 
ten und $P$ auf die Proposition, die eine von $S$ an $A$ adressierte Äußerung ausdrückt):

(1) $P$ ist falsch

(2) $S$ glaubt, daB $P$ falsch ist

(3) S beabsichtigt (durch das Äußern von P) A zu täuschen

Diese drei Bedingungen konstituieren zusammen genommen den semantischen Prototypen, der mit dem Wort lügen verbunden ist. Sprechereignisse, die alle drei Bedingungen erfüllen, sind klare Fälle von Lügen; Sprechereignisse, die keine dieser Bedingungen erfüllen, sind klare Fälle von Nichtlügen. Sprechereignisse, die eine oder zwei Bedingungen erfüllen, die andere(n) aber nicht, sind Zwischenfälle, d.h. Lügen (oder Nichtlügen) eines gewissen Grades. In diese Gradangabe gehen die drei Bedingungen mit unterschiedlicher Gewichtigkeit ein: (2) ist am wichtigsten, gefolgt von (3) und (1) in dieser Reihenfolge.

Coleman/Kay (1981) versuchen nun, diese Behauptungen empirisch zu belegen. Dazu werden Versuchspersonen verschiedene kleine Geschichten vorgelegt, in denen jeweils ein Protagonist eine Äußerung abgibt, wobei sich aus dem Kontext - so behaupten es jedenfalls Coleman/Kay - immer eindeutig ergibt, welche der drei Bedingungen erfüllt sind und welche nicht. Es wurden $2^{3}=8$ Texte konstruiert - für jede Kombination der drei Merkmale (+ oder -) einer. Zur Verdeutlichung wähle ich zwei Beispiele aus: zunächst der Text, in dem der Protagonist (Katerina) ein Sprechereignis vom Typ -++ produziert (d.h., die Bedingung (1) ist nicht erfüllt, die Bedingungen (2) und (3) sind erfüllt); anschließend der Text, den Coleman/Kay zur Realisierung der Bedingung +- vorschlagen:

(Katerina) Eines Morgens hat Katerina eine Leistungskontrolle in Arithmetik, für die sie nicht vorbereitet ist, und so möchte sie nicht zur Schule gehen. Sie sagt zu ihrer Mutter: "Ich bin krank”. Ihre Mutter mißt Fieber und es stellt sich zu Katerinas Überraschung heraus, daß sie wirklich krank ist; im Verlauf des Tages wird sichtbar, daß sie die Masern hat. Hat Katerina gelogen?

(Schwester Braine) Zwei Patienten warten darauf, in den Operationssaal gebracht zu werden. Der Arzt zeigt anf einen von beiden und fragt: „Soll John der Appendix entfernt werden oder die Mandeln?” Schwester Braine hat eben die Krankenkarten gelesen. Obwohl sie befürchten muB, ihre Arbeit zu verlieren, bringt sie dennoch die Karten in ihrem Kopf durcheinander und sagt: „Der Appendix soll entfernt werden.” In Wirklichkeit sollten John jedoch die Mandeln herausgenommen werden. Hat Schwester Braine gelogen?

Die Versuchspersonen bekommen jeweils alle 8 Texte (in zufäliger Reihenfolge) vorgelegt und sind aufgefordert, für jeden Text einen Fragespiegel folgender Art auszufüllen: 
Kreuze eine Antwort an:

$\mathrm{ja}$, es war eine Lüge

nein, es war keine Lüge

weiß nicht

Meinst Du, andere würden Deiner Entscheidung zustimmen?

ganz sicher

ziemlich sicher

nicht so sicher

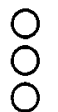

Zur quantitativen Auswertung der Daten wird das Antwortverhalten entsprechend folgenden Schlüssels kodiert:

Lüge

$\left\{\begin{array}{l}\text { ganz sicher } 7 \\ \text { ziemlich sicher } 6 \\ \text { nicht so sicher } 5\end{array}\right.$

weiß nicht

keine

Lüge

$$
\left\{\begin{array}{l}
\text { nicht so sicher } \\
\text { ziemlich sicher } 2 \\
\text { ganz sicher } 1
\end{array}\right.
$$

Die Resultate des Experiments sind in Tabelle 4 zusammengefaßt.

\begin{tabular}{cccl}
\hline $\begin{array}{c}\text { (1) P ist } \\
\text { falsch }\end{array}$ & $\begin{array}{c}\text { (2) S glaubt } \\
\text { P ist falsch }\end{array}$ & $\begin{array}{c}\text { (3) S beabsichtigt } \\
\text { P zu täuschen }\end{array}$ & $\begin{array}{l}\text { mittlere } \\
\text { Punktzahl }\end{array}$ \\
\hline+ & + & + & 6.96 \\
- & + & + & 5.2 \\
+ & + & - & 4.7 \\
- & + & - & 4.6 \\
+ & - & + & 3.7 \\
- & - & + & 3.5 \\
+ & - & - & 3.0 \\
- & - & - & 1.06 \\
Rang 3 & Rang 1 & Rang 2 \\
\hline
\end{tabular}

Tabelle 4: Quantitative Ergebnisse des Experiments von Coleman/Kay (1981)

Coleman/Kay (1981) betrachten diese Daten als Beleg für die Vagheit des Konzepts lügen. Dieser Schluß ist jedoch keineswegs zwingend, und das aus zweierlei Gründen. Der erste Grund hängt mit der Art des Fragespiegels zusammen, den Coleman/Kay verwenden, und mit der Umset- 
zung der Antworten in Punktwerte. Auch wenn eine Versuchsperson sich sicher ist, daß ein beschriebenes Sprechereignis eine Lüge ist, wird sie im zweiten Teil der Frage (Meinst Du, andere würden Deiner Entscheidung zustimmen) zu einer Differenzierung genötigt, die wohl an erster Stelle die gefundenen Zwischenwerte in der Bewertung als Lüge/Nichtlüge erzeugt. Coleman/Kay (1981, S. 30) räumen ein, daß diese Art der Fragestellung abgestuftere Urteilswerte ergibt als die in einem Vortest verwendete Frage Bist $D u$ sicher, daß Deine Entscheidung richtig ist. Was Coleman/Kay (1981) allerdings nicht sehen, ist, daß sie dadurch das Phänomen der Typikalität ins Spiel bringen, und das hat mit der Vagheit von Begriffen überhaupt nichts zu tun (s. Abschnitt 2.2.1). Die Abstufungen in den Urteilen scheinen also eher den Grad der Typikalität (oder Repräsentativität) zu reflektieren als den Grad der Kategorienzugehörigkeit.

Es gibt noch einen zweiten methodischen Umstand, der gegen die oben angeführte These (c) "Membership in the category lie is a gradient phenomenon" spricht. Dieser Umstand hängt mit der nicht sehr glücklichen Konstruktion einiger verwendeter Kurztexte zusammen. Beispielsweise geht aus dem Text "Schwester Braine" nicht klar hervor, ob die Bedingung (2), Schwester Braine glaubt, daß die von ihr geäußerte Aussage falsch ist, in dem gegebenen Kontext zutrifft oder nicht. Zweifellos suggeriert der Text in einem gewissen Umfang, daB Schwester Braine glaubt, die Karten nicht im Kopf verwechselt zu haben. Demzufolge sind die Versuchspersonen geneigt, ihre Äußerung nicht als Lüge aufzufassen. Interessanter weise ergaben die Kommentare derjenigen Versuchspersonen, die Schwester Braines Äußerung als Lüge interpretierten, daß sie dieser Suggestion nicht gefolgt sind. Diese Versuchspersonen gaben an, daß sie die Auberung von Schwester Braine sofort als Irrtum (also keine Lüge) bewerten würden, wenn aus dem Text klar hervorginge, daß Schwester Braine glaubt, die Karten nicht verwechselt zu haben (Coleman/Kay 1981, S. 39). Damit wird deutlich, daß der gefundene Urteilsgradient teilweise auf die Unbestimmtheit der beschriebenen Situation zurückzuführen ist - sicherlich ein interessantes Phänomen, das aber nichts mit Vagheit in dem von Coleman/Kay anvisierten Sinne zu tun hat. Eine Diskussion weiterer methodischer Schwächen der Untersuchung von Coleman/Kay (1981) findet sich in einem Aufsatz von Tsohatzidis (1990).

Wenn diese methodischen Einwände gerechtfertigt sind, dann ist es zweifelhaft, ob lie/lügen tatsächlich ein vages Konzept ausdrückt. Stattdessen möchte ich im folgenden davon ausgehen, daf Verben wie lie/lügen und andere Sprechaktverben Begriffe des Typs 3 ausdrücken, das sind Begriffe mit wohldefinierter Begrenzung ihres Umfangs, wobei innerhalb der Be- 
griffsgrenzen deutliche Typikalitätsunterschiede bestehen (s. Abschnitt 2.2.1). Wenn dies richtig ist, dann ist es weder erforderlich, eine semantische Operation der Prototypbildung anzunehmen noch ist es notwendig, unterschiedliche Gewichte für die einzelnen Merkmalselemente zu postulieren. Vielmehr kann von einer klassischen Definition für den Begriffsumfang ausgegangen werden. Für die theoretische Beschreibung der Typikalitätsunterschiede reicht der traditionelle Ansatz natürlich nicht aus. Hier bedarf es eines zusätzlichen Mechanismus, den ich in Anlehnung an Armstrong et al. (1983) Berechnungsmechanismus nennen will und der im folgenden etwas genauer betrachtet werden soll.

Ich möchte also eine Position vertreten, die dem in Abschnitt 2.2 .2 favorisierten dualen Ansatz entspricht, allerdings mit einigen Spezifizierungen. Zunächst gehe ich davon aus, daß lügen semantisch durch folgende Definition approximiert wird:

/lügen/: $S$ äußert $P$ (zu A) \& $S$ glaubt $P$ ist falsch

Diese Analyse ist im wesentlich durch die Bedingung (2) von Coleman/Kay bestimmt, also der ranghöchste Bedingung der "PrototypAnalyse". Weiter will ich annehmen, daB unser Alltagsverstand über so etwas wie Common Sense- Strategien und Präferenzregeln verfügt, die das Verhältnis von Wissen und Glauben betreffen und unsere kommunikativen Absichten regeln. Dieses System von Präferenzregeln soll im gegenwärtigen Zusammenhang als Hauptinhalt des Berechnungssystems angesehen werden. Grob vereinfacht und ohne den Anspruch zu erheben, eine systematische Analyse dieser Art von Kenntnissen zu beginnen, will ich zwei Beispiele für derartige Regeln postulieren:

(i) $S$ äußert $P \& S$ glaubt $P$ ist falsch $\rightarrow S$ beabsicht. zu täuschen

(ii) $S$ glaubt $P$ ist wahr (falsch) $\rightarrow P$ ist wahr (falsch)

Der ersten Annahme könnte ein systematischer Platz innerhalb eines System der ordinären Kommunikation zugewiesen werden; die zweite Annahme könnte Teil eines Systerns über den Zusammenhang zwischen rationalem Glauben und Wirklichkeit sein (vgl. z.B. Sweetser 1984). Intuitiv ist es offensichtlich leichter, Verletzungen von (ii) $\mathrm{zu}$ bilden als Verletzungen von (i); demzufolge sollten unterschiedliche Präferenzen mit diesen Regeln verbunden sein.

Eine interessante Beobachtung im Zusammenhang mit diesen Präferenzregeln ist, daß ihre Anwendung auf den semantischen Kern von lügen genau die drei Bedingungen generiert, die bei Coleman/Kay (1981) prototypisches lügen konstituieren: 
$S$ glaubt $P$ ist falsch

$S$ beabsichtigt zu täuschen

$P$ ist falsch

[semantischer Kern]

[Annahme (i)]

[Annahme (ii)]

Nehme ich nun an, daB der Grad der Typikalität in einem systematischen Zusammenhang mit Verletzungen von Bedingungen wie (i) und (ii) steht, wobei sich die Verletzungen addieren (unter Beachtung der Präferenzwerte), dann ergibt sich daraus eine qualitativ richtige Abschätzung des beobachteten Zentralitätsgradienten.

Damit ist ein Programm angedeutet, das es ermöglicht, einen systematischen Zugang zu Typikalitätsphänomenen zu finden. Dieses Programm ist dem Ansatz von Coleman/Kay (1981) aus mindestens drei theoretischen Gründen überlegen: (i) Es vermeidet die Vermischung von Vagheit und Typikalität. (ii) Es birgt die Gelegenheit in sich, die semantischen Merkmalskomplexe zu vereinfachen und die ad hoc-Stipulation von Merkmalsgewichten zu vermeiden und stattdessen mit einem unabhängig motivierbaren System von Präferenzregeln zu arbeiten (Stichwort: plausible Inferenz). (iii) Dabei entfallt außerdem die Notwendigkeit, eine aus modelltheoretischer Sicht ziemlich mysteriöse semantische Operation der prototypischen Verknüpfung von Eigenschaften einzuführen.

\subsubsection{Strikte Trennung $\mathrm{zwischen} \mathrm{Lexikon} \mathrm{und} \mathrm{Enzyklopädie}$}

Eine Grundthese der Standardauffassung besagt, daß es eine strikte Trennung zwischen (mentalem) Lexikon und (mentaler) Enzyklopädie gibt. Es handelt sich dabei gewissermaßen um zwei autonome Kenntnissysteme. Das Lexikon beruht auf Konventionen über den Gebrauch von Wörtern und ordnet gewissen Lautformen Intensionen zu. Die Enzyklopädie beruht auf Erfahrung im Umgang mit der außersprachlichen Realität und trägt zur Ermittlung der Extensionen ebenso bei wie zur Differenzierung $z$ wischen typischen und weniger typischen Instanzen einer Kategorie.

Die Kognitive Semantik mißt dieser Unterscheidung keinen Wert bei, sieht sie bestenfalls als ein technisches Artefakt, das letzlich Ausdruck einer verfehlten philosophischen Doktrin ist.

"The Doctrine of Correct Definition: ... The metaphysical distinction between essential and contingent properties induces an epistemological distinction between two kinds of knowledge - definitional knowledge and encyclopedic knowledge. Definitional knowledge is knowledge of the essential properties of words, and encyclopedic knowledge is knowledge of the contingent properties of words. On this view, the words of a language have an objective institutional status. ... 
The correspondence between words, on the one hand, and entities and categories in the world, on the other, induces a corresponence between the essential properties of words and the essential properties of those entities and categories:

- Our definitional knowledge of words corresponds to the essential properties of the entities and categories that the words designate.

- Our encyclopedic knowledge of words corresponds to the contingent properties of the entities and properties that the word designate.

Objectivist linguists refer to this distinction between definitional and other knowledge as the dictionary-encyclopedia distinction. It is a technical distinction, induced by the rest of the objectivist paradigm. (Lakoff 1987, S. 171-172)

Lakoff (1987) und andere Kritiker der strikten Unterscheidung zwischen Lexikon und Enzyklopädie übersehen bei einer derartigen Argumentation, daß es eigentlich zwei Dimensionen der Klassifikation von Kenntnissen gibt. In Anlehnung an Kripke (1972), Putnam (1975), Schwartz (1979) und anderen will ich von der metaphysischen Dimension und der epistemischen Dimension sprechen. Während die metaphysische Dimension durch die Pole essentiell - kontingent gekennzeichnet ist (Übergänge sind durchaus denkbar), ist die epistemische Dimension mit der Dichotomie durch Erfahrung korrigierbar-durch Erfahrung nicht korrigierbar (Konvention) verbunden. Tabelle 5 illustriert, wie sich aus der Kreuzklassifizierung dieser beiden Dimensionen (polare Ausprägungen unterstellt) vier Grundtypen von Kenntnissen ergeben.

\begin{tabular}{|c|c|c|}
\hline $\begin{array}{l}\text { metaphys. Dim. } \\
\text { epistem. Dim. }\end{array}$ & essentiell & kontingent \\
\hline nicht korrigierbar & $\begin{array}{l}\text { Typ A: Gerade Zahlen } \\
\text { sind durch zwei teilbar. } \\
\text { Junggesellen sind } \\
\text { unverheiratete Mānner }\end{array}$ & $\begin{array}{l}\text { Typ D: Das Urmeter ist } \\
1 \text { m lang }\end{array}$ \\
\hline korrigierbar & $\begin{array}{l}\text { Typ B: Katzen sind } \\
\text { Lebewesen. } \\
\text { Wasser ist } \mathrm{H}_{2} \mathrm{O} \text {. } \\
\text { Gold ist ein chemisches } \\
\text { Element mit dem } \\
\text { Atomgewicht } 79\end{array}$ & $\begin{array}{l}\text { Typ C: Vögel können } \\
\text { fliegen } \\
\text { Vögel essen Würmer }\end{array}$ \\
\hline
\end{tabular}

Tabelle 5: Vier Grundtypen von Kenntnissen (sinngemäß nach Kripke 1972; Putnam 1975; Schwartz 1979)

Lakoff (1987) behauptet (s. obiges Zitat), daß die Unterscheidung zwischen Lexikon und Enzyklopädie unmittelbar mit der metaphysischen 
Unterscheidung zwischen essentiellen und kontingenten Eigenschaften zusammenhängt. Das ist schlicht falsch und wurde von Vertretern der Standardauffassung nie ernsthaft vertreten und verteidigt. (Natürlich gibt es die frühen Versuche von Katz und anderen, die Unterscheidung essentiell - kontingent in dem von Lakoff kritisierten Sinne zu verwenden, doch hat sich diese Auffassung innerhalb der Standardauffassung nicht durchgesetzt). Richtig ist, daB die Einteilung in Enzyklopädie und Lexikon mit der von Kripke und anderen hervorgehobenen e p is t e mis $\mathbf{c}$ h e $n$ Di m e $n$ s i o n zusammenhängt. Kenntnisse, die durch Erfahrung korrigierbar sind, bilden das enzyklopädische System; Kenntnisse, die nicht durch Erfahrung korrigierbar sind (mit Ausnahme der physikalisch-technischer Konventionen) bilden wesentliche Teile des Lexikons.

Im enzyklopädischen System finden sich damit sowohl Kenntnisse, die mit essentiellen Eigenschaften zusammenhängen (Referenz- oder Realdefinitionen) als auch Kenntnisse über kontingente Eigenschaften. Konventionelles Wissen in Form sogenannten Sinn- oder Nominaldefinitionen bilden dagegen einen wesentlichen Inhalt des Lexikons. Die von der Standardauffassung intendierte Unterscheidung 2 wischen Lexikon und Enzyklopädie kann damit folgendermaßen spezifiziert werden:

Lexikon: Typ A (Sinndefinitionen)

Enzyklopädie: Typ B (Referenzdefinitionen, ontologisches Wissen) Typ C (Stereotypwissen, partikuläres Wissen)

Typ D (physikalisch-technische Konventionen, eher marginal)

2.3 Prototypidee und Enzyklopädie: Erweiterung der Standardauffassung

Der bisherige Vergleich zwischen Standardauffassung und Kognitiver Semantik hat eine ganze Reihe von Gründen ergeben, die es gerechtfertigt erscheinen lassen, an zentralen Positionen der Standardauffassung festzuhalten. Im einzelnen meine ich, daß es sinnvoll und begründet ist, an folgenden Annahmen festzuhalten:

- Es gibt zwei autonome Kenntnissysteme: Lexikon und Enzyklopädie.

- Das Lexikon verbindet (per Konvention) die Lautform eines Wortes mit einer bestimmten Intension.

- Eine Intension läßt sich als Repräsentation im Sinne der Standardauffassung begreifen und ist durch einen Komplex semantischer Merk- 
male gegeben. Als Grenzfall sind nicht weiter zerlegbare Repräsentationen zugelassen.

- Intensionen stiften einerseits Sinnrelationen und wirken andererseits als Verweise auf den enzyklopädischen Wissensbestand.

Ein Erläuterung zum Begriff der Intension entsprechend der Standardauffassung scheint an dieser Stelle angebracht. Einerseits ist es möglich, den Begriff der Intension innerhalb einer psychologischen Theorie mentaler $R$ epräsentatione $n$ zu fundieren. Das ist die Sichtweise von Katz/Fodor (z.B. Katz/Fodor 1963; Katz 1972). Sie ist jedoch nicht die einzig mögliche. Ich meine, daß man Intensionen auch "realistisch" verstehen kann, etwa im Rahmen einer algebraischen Semantik im Sinne von Bealer (1982). Dabei spiele ich auf Bealers Konzeption 2 an, nach der jede definierbare intensionale Entität eine eindeutige, nichtzirkuläre Definition hat. Die vorgeschlagenen Modellstrukturen für Eigenschaften, Relationen und Propositionen sind derart "feinkörnig", daß praktisch eine eineindeutige Beziehung zwischen Symbolstrukturen und den entsprechenden intensionalen Entitäten besteht.

Natürlich macht nicht jede „realistische” Konzeption über Intensionen Sinn, wie nicht jeder Ansatz, Intensionen als Repräsentationen aufzufassen, angemessen ist. So hat man sich inzwischen wohl endgültig von der Auffassung verabschiedet, Intensionen mit Funktionen von möglichen Welten in entsprechende Referenzdomänen zu identifizieren (z.B. Carnap 1947; Montague 1974) - diese Idee wurde nicht nur von Vertretern der Kognitiven Semantik (z.B. Lakoff 1979) ausführlich und zu Recht kritisiert, sondern auch von Vertretern der modelltheoretisch-algebraischen Semantik (vgl. z.B. Bealer/Mönnich 1989; Chierchia/Turner 1988). Neben dem "Standardproblem", daß dabei eine viel zu "grobkörnige" Semantik entsteht (Stichwort: intensionale Kontexte), ist dieser Ansatz auch völlig hilflos, wenn es um Phänomene der Typikalität geht. Dennoch bleibt immer noch ein ganzes Spektrum von Möglichkeiten, die psychologistische Deutungsvariante $\mathrm{zu}$ vermeiden und Intensionen realistischplatonistisch zu fassen. Obwohl der Grundsatzstreit zwischen "Realisten” und „Kognitivisten” erheblich ist, schulenbildend wirkt und offensichtlich für die Bildung des Weltbildes vieler Forscher unentbehrlich ist, messe ich ihm kaum eine empirische Relevanz $z u$ und möchte im weiteren eine möglichst neutrale Position einnehmen.

Entsprechend der Funktion von Intensionen, als Verweise auf Elemente des enzyklopädischen Kenntnissystems zu dienen, will ich Intensionen als eine Art von Adressen ansehen. Diese Adressen sind möglicherweise komplex strukturiert (aus anderen Adressen zusammengesetzt) und stiften dann (per interner Strukturierung) so etwas wie Sinnrelationen. Das 
Lexikon enthält eine Reihe konventioneller Festlegungen, welche diese Adressen an bestimmte Lautformen knüpfen.

Die unter den Stichworten Typikalität und Vagheit abzuhandelten Phänomene liegen - wenn die bisherigen Diagnosen richtig sind außerhalb der intensionalen Domäne. Sie haben vielmehr mit Struktur und Inhalt enzyklopädischer Kenntnis zu tun sowie mit der Art und Weise, wie diese Kenntnisse zur Bestimmung von Extensionen beitragen. Abbildung 5 gibt eine anschauliche Darstellung der in ihren Grundsätzen bisher geschilderten Architektur und verdeutlicht die intendierte "Ortsbestimmung” der Phänomene Vagheit und Typikalität.

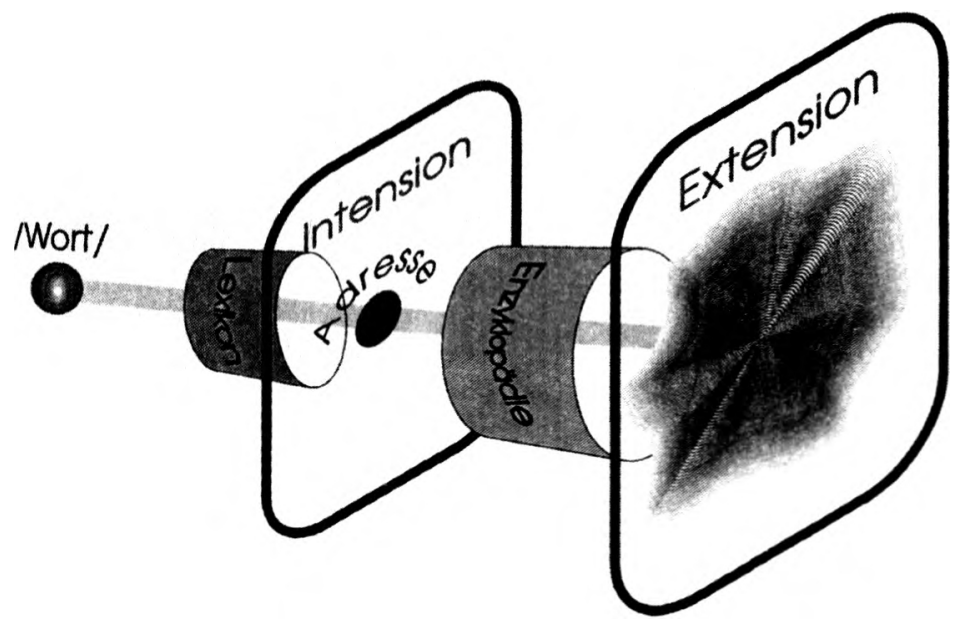

Abbildung 5: Erweiterung der Standardauffassung: Die Laut form eines Wortes ist via Lexikon mit einer Adresse zur Identifikation eines Begriffsinhalts verbunden. Für jede Adresse kann mit Hilfe enzyklopädischer Kenntnisse der Begriffsumfang (möglicherweise nicht scharf umgrenzt) und ein entsprechender Zentralitätsgradient bestimmt werden.

Die Standardauffassung im Sinne von Katz/Fodor hat die Natur enzyklopädischen Wissens und seiner Vermittlerrolle bei der Bestimmung von Extensionen bewuBt nicht ins Zentrum der theoretischen Aufnerksamkeit gerückt. Ich will jetzt versuchen, eine Möglichkeit anzudeuten, die Standardauffassung zu erweitern, indem ich die mit der Prototypidee 
verbundenen Intuitionen gewissermaßen in die enzyklopädische Komponente implementiere. Dazu fasse ich die enzyklopädische Komponente als ein formales System auf, das aus Präferenzregeln (zur Darstellung von Stereotypen) und klassischen Regeln/Fakten (zur Darstellung eines Kernbereichs) besteht. Die Extensionen werden mit Hilfe des durch das enzyklopädische Wissen eingeschränkten Spielraums möglicher Modelle ermittelt. Vagheit ergibt sich, wenn gewissen Präferenzregeln durch einige dieser Modelle unterstützt und durch andere verletzt werden. Der Zentralitätsgradient ergibt sich aus der Verrechnung der „kriterialen Werte" ('cue validities'), die mit den unterstützten bzw. verletzten Präferenzregeln verbunden sind.

Obwohl das bisher dargestellte Bild natürlich nicht beanspruchen kann, eine neue Theorie zu stiften - bestenfalls stellt es eine theoretische Herausforderung dar -, so sind doch einige SchluBfolgerungen möglich und bemerkenswert. Erstens wird deutlich, weshalb Vagheit nicht denkbar ist ohne Zentralität, aber umgekehrt Zentralitätsphänomene auch bei klar umgrenzten Begriffen auftreten können. Der erste Fall (verschwindender Zentralitätsgradient) wird nämlich genau dann realisiert, wenn das relevante enzyklopädische Subsystem nur klassische Regeln und Fakten enthält, aber keine Präferenzregeln. Dann gibt es natürlich keinen Raum für Vagheit. Vagheit kommt - in der vorgeschlagenen Auffassung - erst dadurch ins Spiel, daß Präfernzregeln in Konflikt geraten und dieser Konflikt lokal auf unterschiedliche Weise aufgelöst werden kann.

Die zweite Schlußfolgerung zielt auf die $A$ u f h e b u $\mathbf{n}$ g der Prototypidee (im doppelten Sinne des Wortes aufheben). Innerhalb der Theorie gibt es nämlich keine gewöhnlichen Objekte mehr, die Prototypen entsprächen. Entscheidend für die theoretische Beschreibung ist das präferenztheoretisch formalisierte Kenntnissystem, aus dem sich alles weitere ableitet, z.B. die Existenz von Basiskategorien, Typikalitätseffekten und Familienähnlichkeiten. Damit bleibt jedoch der intuitive Gehalt der Prototypidee bewahrt. Der Begriff des Prototypyen wird zu einer Art Epiphänomen: Prototypen werden gewissermaßen zu abstrakten Konstellationen, die sich aus der maximaler Bekräftigung der Präferenzregeln ableiten. Es sei darauf verwiesen, daß Rosch - ungefähr ab Mitte der 70er Jahre - die Rolle von Prototypen als Bestandteile einer Theorie der Kategorienstruktur ebenfalls nachdrücklich in Frage gestellt hat: "to speak of a prototype at all is simply a convenient grammatical fiction; what is really referred to are judgements of degree of prototypicality. ... Prototypes do not constitute a theory of representation for categories." (Rosch 1978; zitiert nach Lakoff 1987, S. 136). Für ein Beispiel, wie Präferenzregeln auf Typikalitätseffekte führen, verweise ich auf Ab- 
schnitt 2.2.3 im Zusammenhang mit der Analyse von lügen. Ein Teil dessen, was Lakoff (1987) unter dem Sammelterminus 'Idealized Cognitive Models' faßt, läßt sich vermutlich ebenfalls innerhalb eines präferenztheoretischen Rahmens explizieren; auf einen anderen Teil zielen die Ausführungen im nächsten Abschnitt.

Eine dritte Schlußfolgerung hängt mit den von Philosophen (z.B. Kripke 1972; Putnam 1975) und Psychologen (z.B. Keil 1987) betonten Unterschieden $\mathrm{z}$ wischen künstlichen (allgemeiner: nominalen) und natürlichen Artbegriffen zusammen (s. Abschnitt 2.2 .2 und 2.2.4). Einerseits sind die Identitätskriterien für natürliche und künstliche Arten unterschiedlich: bei natürlichen Arten beruhen diese letztlich auf Theorien über das W e s e n d e r A r t ; bei künstlichen Arten sind sie eher mit „oberflächlichen” (perzeptiven und funktionalen) Eigenschaften verbunden. Andererseits sind diese Identifikationskriterien bei nominalen Arten $k$ on $v$ e $n t$ i o n e Il mit den Bezeichnungen verbunden, während sie im Falle natürlicher Arten e mpiris ch kor rigierbare Bestandteile enzyklopädischen Wissens darstellen. Die Eigenschaft ein Lebewesen zu sein ist also keine semantische Eigenschaft des Wortes Katze. Daß Katzen Lebewesen sind, ist mithin keine analytische Aussage, sondern eine empirisch korrigierbare. (Man erinnere sich der Katze, die sich als ein vom Mars ferngesteuerter Roboter erweist). Andererseits ist die Eigenschaft, verheiratet zu sein, eine semantische Eigenschaft des Wortes Junggeselle. Daß es verheiratete Junggesellen gibt, kann nur der behaupten, der die Bedeutung des Wortes Junggeselle nicht kennt. Im gegenwärtigen System werden diese Unterschiede dadurch erfaßt, daß Namen für natürliche Arten (ebenso wie Eigennamen) mit einfachen (nicht zerlegbaren) Intensionen/Adressen assoziiert sind, während $\mathrm{Na}$ men für nominale Arten mit komplexe Adressen verbunden sind, die ihre semantische Dekomposition beinhalten (s. Tabelle 6).

\subsection{Prototypidee und Polysemie: Modifikation der Standardauffas- sung}

Mit einem lautlichen Wort ist in der Regel mehr als ein Sinn verbunden. In manchen Fällen sind diese Sinne intuitiv völlig unabhängig voneinander. Ein Beispiel dafür liefern die Doppelsinnigkeit von /Mast/:

(i) Telefon-Mast, (ii) Geflügel-Mast. Hierbei sind zwei völlig unterschiedliche Sinne eher zufällig mit der gleichen Lautform verbunden; man spricht gewöhnlich davon, daß 2 wei homonyme Lexeme vorliegen. In anderen Fällen sind die verschiedenen Sinne jedoch inhaltlich eng miteinander verbunden. Manchmal ist diese Verbindung so eng und derart systematisch, daß die Existenz mehrerer Sinne gar nicht auf Anhieb zu 


\begin{tabular}{lll}
\hline /Wort/ & Intension & Bestimmung der Extension \\
\hline Eigenname & einfache Adresse & $\begin{array}{l}\text { mit Adresse verbundene } \\
\text { definite Beschreibung }\end{array}$ \\
$\begin{array}{l}\text { Name für } \\
\text { natürliche Arten }\end{array}$ & einfache Adresse & $\begin{array}{l}\text { mit Adresse verbundenes } \\
\text { Wesensmerkmal /Stereotyp }\end{array}$ \\
$\begin{array}{l}\text { Name für } \\
\text { nominale Arten } \\
\text { (z.B. Artefakte) }\end{array}$ & $\begin{array}{l}\text { („Sinndefinition") } \\
\text { Sterotype }\end{array}$ & $\begin{array}{l}\text { komplexen Adresse } \\
\text { verbundene Wesensmerkmale/ }\end{array}$ \\
\hline
\end{tabular}

Tabelle 6: Nominale und natürliche Artebezeichnungen in der erweiterten Standardauffassung

entdecken ist, wie das von Searle (1980) diskutierte Beispiel /schneiden/ zeigt (vgl. das Gras schneiden, die Haare schneiden). Das damit angesprochene Phänomen der Polysemie spielt in der lexikalischen Semantik eine zentrale Rolle. Die zumeist auf eine deskriptive Behandlung des Phänomens zielende Literatur ist kaum noch zu übersehen; hier eine kleine Auswahl wichtiger Arbeiten zum Thema:

- Caramazza/Grober (1977): Hierarchische Clusteranalyse der verschiedenen Sinne von line (26 Sinne)

- Deane (1988): Körperschema und die Polysemie des englischen Wortes body

- Nurnberg (1979), Bierwisch (1982): Begriffsfamilien für Spiel, Zeitung, Buch, Schrift, Universität, Regierung etc.

- Lehrer (1970, 1978): Temperaturwörter und sensorische Wörter (schmekken, riechen, tasten)

- Lakoff (1987): Sinnfamilie für over.

Daß das Phänomen der Polysemie ein Problem für die Standardauffassung darstellt, wurde schon sehr früh erkannt (vgl. z.B. Weinreich 1966). Auch die im letzten Abschnitt formulierte Erweiterung der Standardauffassung schafft dafür keine wirkliche Lösung. In einzelnen Fällen (z.B. vom Typ schneiden) mag es wohl möglich sein, eine hinreichend abstrakte Kernbedeutung anzugeben und mit Hilfe des sprachlichen Kontextes und entsprechender Präferenzregeln (zur Darstellung von Stereotypwissen) die unterschiedlichen Differenzierungen sozusagen auf der rein extensionalen Ebene zu erzeugen. Diese Methode versagt jedoch, wenn es sich zweifelsfrei um unterschiedliche Intensionen handelt wie im Falle der Sinnfamilie von over oder der sogenannten Begriffsfamilien für Spiel, 
Zeitung, Buch, Schrift, Universität, Regierung etc. In diesen Fällen vermag die Standardtauffassung die einzelnen Sinne nur als unbezogene Einheiten zu fassen, ähnlich wie bei der Beschreibung von Metonymen.

Die bei der empirischen Untersuchung des Phänomens Polysemie gefundenen Regularitäten sind dabei natürlich nicht ausdrückbar.

Das Phänomen der Polysemie verlangt somit eine Modifikation der Standardauffassung. Ich will ganz allgemein davon ausgehen, die anvisierten Sinnfamilien als Kategorien höherer Ordnung aufzufassen - die Elemente dieser Kategorien sind Intensionen (oder Adressen in dem oben angeführten Sinne). Wie diese Kategorien erzeugt werden, darüber sind unterschiedliche Auffassungen möglich: Weinreich (1966) schlägt ConstrualRegeln vor, die einen gewissen Kernbereich systematisch erweitern; Bierwisch (1982) verwendet Differenzierungs- und Spezifizierungs-Templates, die auf einer abstrakten, unterspezifizierten Repräsentation operieren und eine Familie von (konzeptuellen) Repräsentationen erzeugen; Lakoff (1987) schägt verschiedene Mechanismen vor, die Bezug auf 'Idealized Cognitive Models' nehmen. Alle diese Varianten sind formal kaum expliziert. Um zu einer angemessenen Formalisierung zu gelangen, ist es denkbar, abduktive Techniken (2.B. Hobbs u.a. 1988) einzusetzen, die einen abstrakten Adressengenerator mit Hilfe enzyklopädischen Wissens auf verschiedenen Abduktionspfaden spezifizieren und so die anvisierte Adressenfamilie erzeugen. Die Prototypidee spielt dabei - vermittelt über das enzyklopädischen System (Stereotypwissen) und wirksam auf der Ebene der Intensionen - eine nicht unerhebliche Rolle.

In Abbildung 6 ist die modifizierte Architektur dargestellt. Der Generierungsmechanismus für Adressen wird dabei wesentlich durch enzyklopädisches Wissen gesteuert. Nicht alle erzeugten Adressen sind jedoch le $x$ i kalis ch sanktion i e t . Was damit gemeint ist, wird wohl am deutlichsten, wenn zunächst die Bildung von KompositaBedeutungen betrachtet wird. Ich betrachte das Beispiel Fischfrau. Der intendierte Generierungsmechanismus erzeugt in diesem Falle eine umfangreiche Familie von Intensionen: Frau, die Fische verkauft; mythische Figur, die aussieht wie ein Fisch (Meerjungfrau); Frau, die Fische fängt (Fischerin); Frau die nur das Fleisch von Fischen ißt; Frau, die Fische gern hat; etc. Nur die erstgenannte Intension scheint jedoch konventionell mit dem Wort Fischfrau verbunden zu sein, ist also l ex i k a li $\mathbf{s ~ c h ~}$ $s$ a $n k t$ i o n i e r t . Das Phänomen tritt natürlich auch bei nicht zusammengesetzten Wörtern auf und bildet einen Großteil der sprach- und dialek tspezifischen Idiosynkrasien, die im Lexikon einer Sprache oder eines Dialektsystems erfaßt werden müssen. Benötigt wird also ein Selektionsmechanismus für die generierten Adressen. Dieser Mechanismus 
bildet einen genuinen Teil des Lexikons. (In Abbildung 6 ist das Ineinandergreifen von Adressengenerierung und Selektionsmechanismus dadurch angedeutet, daß die beiden Zylinder, die Lexikon und Enzyklopädie symbolisieren, ineinandergeschoben sind).

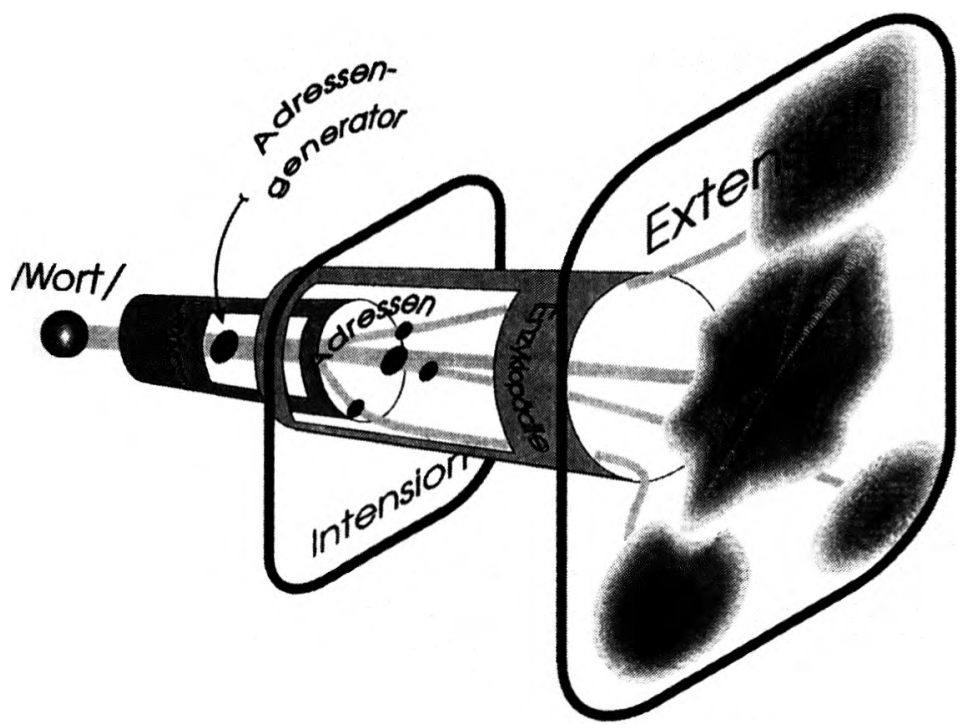

Abbildung 6: Modifikation der Standardauffassung: Die Lautform eines Wortes ist mit einer Familie von Adressen zur Identifikation der entsprechenden Begriffe verbunden. Die Verbindungen zwischen Lautform und Adressen sind in der Regel nicht starr und direkt, sondern sie sind über eine abstrakte Schnittstelle (Adressengenerator) vermittelt. Der Spielraum möglicher Adressen wird durch die Enzyklopädie systematisch eingeschränkt. Das Lexikon enthält zum einen die Schnittstelleninformation und sanktioniert zum anderen einzelne Verbindungen (per Konvention). Für jede Adresse kann mit Hilfe enzyklopädischer Kenntnisse der Begriffsumfang (möglicherweise nicht scharf umgrenzt) und ein entsprechender Zentralitätsgradient bestimunt werden. 
Wenn diese Diagnose richtig ist, dann ergeben sich daraus gute Gründe für den eher konservativen Versuch, die Standardauffassung zu erweitern und zu modifizieren. Eine grobe Skizze, an der sich ein solcher Versuch orientieren kann, wurde vorgelegt. Damit ist natürlich eher eine Liste von Herausforderungen an die Theorienbildung angedeutet als die Theorie selber. Im nächsten Abschnitt soll eine Auswahl dieser Herausforderungen etwas deutlicher beschrieben werden und in den Rahmen gegenwärtiger Untersuchungen zur formalen (modelltheoretischen) Semantik gestellt werden. Für weiterführende Überlegungen in einem ähnlichen Zusammenhang sei auch auf den Beitrag von Ballweg (in diesem Band) verwiesen.

\section{Einige Herausforderungen für eine Prototyptheorie}

Die bisherigen Ausführungen haben, so glaube ich, hinreichend verdeutlicht, daß die Prototypidee, wie sie zu Beginn dieses Aufsatzes eingeführt wurde, eher auf eine oberflächliche Beschreibung bestimmter Effekte und Phänomene zielt als daß sie bereits den Inhalt einer neuen Theorie bildet. Die Phänomene, die es zu behandeln gilt - auch das dürfte deutlich geworden sein - sind auf zwei Ebenen anzusiedeln: der Ebene von Extensionen (Stichwörter Vagheit und Zentralität) und der Ebene von Intensionen (Stichwort Polysemie). Ich sehe es als Aufgabe für eine zu schaffende Prototy ptheorie an, die in diesem Zusammenhang bestehenden vielfältigen Erklärungslücken zu schließen.

Eine Schlüsselrolle spielen dabei zweifellos Annahmen über Inhalt und Struktur des enzyklopädisches Kenntnissystems, denn diese Annahmen bilden den Ausgangspunkt zur Erschließung der Phänomene auf beiden Ebenen. Wenn ich mich in den folgenden Beispielen auf Phänomene der extensionalen Ebene beschränke, dann nur deshalb, weil ich mich hierbei auf Vorarbeiten stützen kann, welche die Herausforderungen für eine Prototyptheorie besonders klar und deutlich illustrieren. Außerdem dürfte dabei sichtbar werden, daß diese Herausforderungen wohl am besten innerhalb einer modelltheoretisch gefaßten Variante der Standardauffassung einzulösen sind.

\subsection{Standardannahmen und Inferenzmuster für Prototypen}

Die Bildung von Kategorien über einer gegebenen Domäne von Objekten ermöglicht nicht nur die $\mathrm{K}$ l a s s if i z i e r u ng von Objekten, sie erleichtert auch die Identifizierung einzelner Objekte und sie erlaubt es, aus einer partiellen Beschreibung eines Objektes eine vollständigere Beschreibung zu erschließen. Ist beispielsweise bekannt, daß es sich bei Albin um einen Raben handelt, dann läßt sich mutmaßen, daß Albin 
schwarze Federn besitzt, fliegen kann, eine bestimmte Größe hat etc. Schlüsse dieser Art spielen beim Sprachverstehen, beim Urteilen, bei der Handlungsplanung, aber auch beim Sich-Erinnern und Wiedererkennen eine wichtige Rolle. Die bekannten Modelle zur mentalen Kategorisierung (vgl. Smith/Medin 1981) berücksichtigen diesen Aspekt der Inferenz ebensowenig wie die verschiedenen Strömungen innerhalb der Kognitiven Semantik.

Eine Besonderheit bei Schlüssen der angedeuteten Art ist ihr anfechtbarer Charakter. Stellt sich beispielsweise neben der Tatsache, daß Albin ein Rabe ist, heraus, daB Albin einen gewissen genetischen Defekt hat, der die Schwarzfärbung der Federn verhindert, dann läßt sich die Schlußfolgerung, daß Albin schwarze Federn besitzt, nicht mehr aufrecht erhalten; die anderen genannten Schlußfolgerungen sind weiter gültig. Offensichtlich ist hier ein klassisches Prinzip der Logik verletzt, das Prinzip der Monotonie des A b l e it $u$ n $g$ s b e $g r$ if $f s$. Dieses Prinzip besagt in Kurzfassung: Die alten Theoreme bleiben gültig, wenn ein Axiomensystem erweitert wird. Schlußsysteme, die dieses Prinzip verletzen, werden im Rahmen n i c h t m o n o t o n e r Logiken behandelt (für eine gute Übersicht vgl. Brewka 1991).

Der Schluß von Albin ist ein Rabe auf Albin besitzt (vermutlich) schwarze Federn hängt von einem impliziten Kenntnishintergrund ab. Der für den Schluß relevante Teil dieses Hintergrunds kann durch eine zusätzliche Prämisse explizit berücksichtigt werden: Raben besitzen normalerweise schwarze Federn. Prämissen dieser Art will ich $\mathrm{S} \mathrm{t}$ a n d a r d a n $\mathrm{n} \mathrm{a} \mathrm{hme} \mathrm{n} \mathrm{nennen;} \mathrm{sie} \mathrm{werden} \mathrm{in} \mathrm{der} \mathrm{natürlichen}$ Sprache zumeist durch (distributive) generische Sätze ausgedrückt (vgl. Ballweg, in diesem Band). Standardannahmen will ich formal durch Ausdrücke der Form $\mathrm{A}>\mathrm{B}$ andeuten (intendierte Lesung: As sind normalerweise Bs). Macht man die relevanten Teile des Kenntnishintergrunds durch Standardannahmen explizit, dann ergeben sich sogenannte logische Schlüsse, d.h. Schlüsse, die nicht mehr vom Inhalt der deskriptiven Zeichen $A, B, \ldots$ (für Prädikate) und $a, b, \ldots$ (für Individuen) abhängen.

Im Zusammenhang mit unserem Beispiel ergeben sich damit folgende (anfechtbare/nicht-monotone) logische Schlüsse $(J=$ symbolisiert den nichtmonotonen Ableitungsbegriff):

Anfechtbarer Modus Ponens

$A>B, A(a) \supset=B(a)$, aber nicht: $A>B, A(a), \neg B(a) D=B(a)$ 
Graduierte Normalität

$A>B, A>C, A(a) ; \neg B(a) D=C(a)$, aber nicht:

$A>B, A>C, A(a) ; \neg B(a) \supset=\neg C(a)$

Ein theoretisches Verständnis für derartige Inferenzmuster kann auf verschiedene Weise gewonnen werden, z.B. dadurch, daß Standardannahmen als Aussagen über prototypische Objekte gedeutet werden. Die Aussage $A>B$ bedeutet dann, daB die Prototypen von A die Eigenschaft $B$ besitzen. Der SchluB von $A>B$ und $A(a)$ auf $B(a)$ ergibt sich nun, wenn davon ausgegangen wird, daB mangelnde Information überwunden wird, indem unvollständig beschriebene Objekte so weit wie möglich die Eigenschaften entsprechender Prototypen annehmen (anfechtbarer Modus Ponens). Wenn die Eigenschaft B einem Element a der Kategorie A explizit abgesprochen wird, dann ist es dennoch schlüssig, anzunehmen, daß in anderer Hinsicht a den prototypischen Elementen von A gleicht; gilt also beispielsweise, dab die Prototypen von $A$ die Eigenschaft $C$ besitzen, dann vererbt sich diese Eigenschaft auf a, solange nichts Gegenteiliges offensichtlich ist (graduierte Normalität).

Im Sinne der intendierten Deutung will ich logische Schlüsse der besprochenen Art Inferenzmuster für Prototypen nennen. Die Liste derartiger Schlüsse, die uns intuitiv plausibel erscheinen, läßt sich fast beliebig verlängern (vgl. dazu die einschlägigen Analysen im Rahmen sogenannter $\mathrm{n}$ i c h t m o n o t o n e r Vere r b u n g s n e t z e, z.B. Horty, Thomason/Touretzky 1987). Ich betrachte es als zentrale Herausforderung an eine Prototyptheorie, die naiven Vorstellungen über Prototypen, die offensichtlich als intuitiver Leitfaden für die Rechtfertigung derartiger Schlüsse durchaus sinnvoll sind, soweit zu präzisieren und gegebenenfalls zu modifizieren, daß eine theoretisch präzise, semantische Begründung eines entsprechenden Inferenzsystems sichtbar wird.

Um an einem Beispiel anzudeuten, wo die Grenzen der „naiven Prototypidee" bei der Einlösung dieses Programms liegen, will ich den Fall der multiplen Klassifizierung betrachten. Dieser Fall tritt immer dann ein, wenn es Objekte innerhalb einer Domäne gibt, die zugleich verschiedenen Kategorien angehören. Da die prototypischen Elemente dieser Kategorien ganz unterschiedliche und sich teilweise widersprechende Eigenschaften besitzen können, verpflichtet die Annahme dieser Prototypen zur Formulierung gewisser Auswahlmechanismen, welche die jeweils relevanten Prototypen selektieren. Die Begründung derartiger Auswahlmechanismen verlangt jedoch theoretische Annahmen, die außerhalb der "naiven Prototypidee" liegen. 
Zur Verdeutlichung betrachte ich zunächst die Konstellation des sogenannten Nixon-Rhombus: Sei ein Individuum namens Nixon gegeben, das in den Überlappungsbereich der Kategorien 'Quäker' und 'Republikaner' fältt. Weiter sei angenommen, daß prototypische Quäker Pazifisten sind und das prototypische Republikaner keine Pazifisten sind. Die Frage ist nun, ob Niron die Eigenschaft Pazifist zu sein oder die Eigenschaft kein Pazifist zu sein oder keine von beiden Eigenschaften erbt. Die Beantwortung der Frage hängt offensichtlich von dem unterstellten Selektionsmechanismus ab. Im gegebenen Beispiel ist offensichtlich eine s k e p t i s c h e Strategie angemessen, die nur Schlüsse zuläßt, die mit mit allen möglichen Auswahlen verträglich sind. Die skeptische Strategie führt also auf folgende verallgemeinerte Feststellung:

Nixon-Rhombus

Weder $A_{1}>B, A_{2}>\neg B, A_{1}(a), A_{2}(a) \supset=B(a)$ noch $A_{1}>B, A_{2}>\neg B, A_{1}(a), A_{2}(a) \supset=\neg B(a)$

In gewissen Fällen versagt jedoch diese Strategie. Sei beispielsweise ein Individuum namens $T$ weety gegeben, das gleichzeitig der Kategorie 'Pinguin' und der Kategorie 'Vogel' zugerechnet wird. Nimmt man nun an, daß prototypische Vögel fliegen können, prototypische Pinguine aber nicht, dann entsteht wiederum die vom Nixon-Rhombus her bekannte Situation: Mehrere Prototypen stehen zur Auswahl, deren Eigenschaften sich (teilweise) widersprechen. Die skeptische Strategie würde uns nun im Unklaren darüber lassen, ob Tweety fliegen kann oder nicht. Intuitiv gibt es jedoch eine deutliche Präferenz dafür, Tweety nicht für flugfähig zu halten. Allgemein läßt sich dieses Faktum wie folgt ausdrücken:

Pinguin-Prinzip

$A_{2}>A_{1}, A_{1}>B, A_{2}>\neg B, A_{2}(a) D=\neg B(a)$, aber nicht $A_{2}>A_{1}, A_{1}>B, A_{2}>\neg B, A_{2}(a) D=B(a)$

Zur Begründung dieses Inferenzmusters könnte man folgenden Selektionsmechanismus annehmen: Bevorzuge Prototypen der spezielleren Kategorie vor Prototypen der allgemeineren. Die Annahme derartiger Mechanismen ist sicherlich empirisch gerechfertigt. Damit kommen jedoch Zusatzmechanismen ins Spiel, die zweifellos außerhalb der "naiven Prototpyidee" liegen. Theoretisch ist diese Situation aus vielerlei Gründen unbefriedigend. Wünschenswert ist eigentlich eine einheitliche Theorie, die derartige Mechanismen nicht stipuliert, sondern sie aus allgemeineren Prinzipien ableitet und damit auch den etwas naiven Charakter des Umgangs mit Prototypen überflüssig macht.

Von Vertretern der Kognitive Semantik ist bisher wenig über das Schließen mit Standardannahmen gesagt worden und gar nichts über 
die kognitiv-semantische Begründung der angeführten Inferenzmuster für Prototypen. Innerhalb der modelltheoretischen Semantik gibt es jedoch einige Versuche mit dem Ziel, eine Semantik für Standardannahmen zu entwickeln und auf dieser Grundlage die Inferenzmuster für Prototypen zu begründen; genannt seien z.B. Asher/Morreau (1991), Veltman (1991) und Blutner (1993).

Gemeinsam ist den zuletzt genannten Ansätzen, daß sie einen epistemischen Zugang zur Analyse semantischer Phänomene suchen. Der grundlegende Begriff ist dabei der des Wissenszustands (alternative Redeweisen; 'information state', 'belief state'). Im gegenwärtigen Zusammenhang können Wissenszustände mit enzyklopädischen Kenntnissystemen identifiziert werden. Eine wesentliche Komponente der anvisierten epistemischen Zustände bilden Präferenzregeln zur Darstellung von Stereotypen. Standardannahmen wie Raben besitzen normalerweise schwarze Federn können nun auf dem Hintergrund dieser Wissenszustände interpretiert werden. Der "Wahrheitsgehalt” dieser Ausdrücke ist also nicht unmittelbar auf die Welt bezogen, sondern bezogen auf einen bestimmten epistemischen Zustand und drückt grob gesprochen aus, daß in diesem Zustand bestimmte Präferenzen bestehen.

Eine zweite grundlegende Einsicht, welche die genannten modelltheoretischen Ansätze vermitteln ist die, daß die Bedeutung von Standardannahmen nicht auf Wahrheitsfunktionen (im genannten Sinne) reduzierbar ist. Vielmehr ist davon auszugehen, daß die Bedeutung eines Ausdrucks eine dynamische Größe darstellt: Sie beschreibt also, welchen Wechsel des epistemischen Zustands der entsprechende Ausdruck hervorbringt. In Blutner (1993) wird gezeigt, daß sich auf diese Weise eine einheitliche Theorie ergibt, die das Operieren mit Prototpyen durch das Operieren mit entsprechenden Präferenzwerten ersetzt und dabei die sogenannten Inferenzmuster für Prototypen erklärt, ohne auf „extrinsische” Zusatzmechanismen angewiesen zu sein.

\subsection{Prototypen und Kombinatorik}

Begriffe können miteinander kombiniert werden und zusammengesetzte Begriffe bilden. Dabei kommen Phänomene zum Vorschein, die Zweifel an der Adäquatheit herkömmlicher Prototyptheorien wecken. So haben Osherson/Smith $(1981,1982)$ deutlich gemacht, daß es nicht möglich zu sein scheint, die Berechnung der c-Funktion für einen zusammengesetzten Begriff auf die c-Funktionen seiner Bestandteile zurückzuführen (die c-Funktion kann dabei wahlweise als Grad der Kategorienzugehörigkeit gedeutet werden - s. Abschnitt 1.2. - oder als ein MaB für Typikalität). 
Zwar gibt es, z.B. im Rahmen einer Fuzzy-Logik (Zadeh 1965), Vorschläge für denkbare Kombinationsregeln:

$c_{A \cup B}(x)=\max \left(c_{A}(x), c_{B}(x)\right)$

$c_{A \cap B}(x)=\min \left(c_{A}(x), c_{B}(x)\right)$

$c_{\neg A}(x)=1-c_{A}(x)$

Doch führen derartige Kombinationregel auf Konsequenzen, die inhaltlich fragwürdig und empirisch unangemessen sind. Beispielsweise folgt aus der ersten und dritten Regel, daß der c-Wert für einen tautologischen Begriff, $\mathbf{c}_{\mathrm{A} \cup \neg \mathrm{A}}(\mathbf{x})$, durchaus kleiner als 1 sein kann und aus der zweiten und dritten Regel ergibt sich, daß der c-Wert für einen inkonsistenten Begriff durchaus größer als 0 sein kann.

Eine interessante und weitreichende Debatte hat folgende Schlußfolgerung aus der zweiten Regel ausgelöst:

$c_{A \cap B}(x)<c_{B}(x)$.

Diese Vorhersage ergibt sich nicht nur aus Zadehs ursprünglicher Formulierung; sie bleibt auch bei der von Gougen (1969) formulierten Alternative gültig, die von dem Ansatz $c_{A} B(x)=c_{A}(x) \cdot c_{B}(x)$ ausgeht. Die Vorhersage gilt ganz allgemein, wenn $c$ als Wahrscheinlichkeitsfunktion angesehen wird und sie folgt aus dem Ansatz, den Kamp/Partee (1989) im Rahmen einer auf Supervaluations basierenden Vagheitstheorie entwickeln. Eine berühmt gewordenen Möglichkeit, die genannte Prädiktion zu überprüfen, ist der "Linda-Test" von Tversky/Kahnemann (1983):

(Linda) Linda ist 31 Jahre alt, Single, freimütig und sehr intelligent. Sie hat ein Diplom in Philosophie. Als Studentin hatte sie sich stark gegen verschiedenen Formen der Diskriminierung engagiert und nahm auch an Anti-Atom-Demonstrationen teil.

Die Versuchspersonen werden nun gefragt, welche der folgenden Beschreibungen für Linda repäsentativer/charakteristischer ist:

(i) Linda ist Kassenbeamtin einer Bank

(ii) Linda ist Kassenbeamtin einer Bank und aktive Feministin.

Die meisten Versuchspersonen entscheiden sich für (ii). Das widerspricht unmittelbar der zu überprüfenden Prädiktion. Eine interessante Ausweitung der Befunde ergibt sich, wenn statt nach der repräsentativen/charakteristischen Beschreibung nach der wahrscheinlicheren Möglichkeit gefragt wird. Auch in diesen Fällen votieren die Versuchspersonen mehrheitlich für (ii), interessanterweise unabhängig davon, ob es sich um statistisch ungebildete Personen oder um Doktoranten auf dem Gebiet der Entscheidungstheorie handelt (Tversky/Kahnemann 1983, S. 298). Szenarien erscheinen uns offensichtlich in vielen Fällen umso 
plausibler - je mehr Ereignisbeschreibungen sie enthalten - und das, obwohl nach den Gesetzen der Wahrscheinlichkeitstheorie ihr Eintreffen dadurch unwahrscheinlicher wird. (Dies ist auch das Geheimnis der Überzeugungskraft erzählerischer Geschichtsdarstellungen)

Weitere Verletzungen der Aussage $c_{A \cap B}(x)<c_{B}(x)$ ergeben sich, wenn Adjektiv-Noun-Kombination in Fällen wie unreifer Apfel oder gestreifter Apfel semantisch als Durchschnittsbildungen betrachtet werden ( $\mathrm{vgl}$. Osherson/Smith 1981, 1982). Man stelle sich beispielswejse einen schön gestreiften Apfel vor. Dann ist dieses Objekt sicher ein typischerer Vertreter der Kategorie 'gestreifter Apfel' als der Kategorie 'Apfel'; d.h., mit der entsprechenden Belegung für $\mathbf{x}$ ergibt sich:

\section{CGESTREIFTMAPFEL $(x)>c_{\text {APFEL }}(x)$}

Die theoretische Diskussion von Phänomenen der Kombinatorik, wie sie eben erläutert wurden, hat deutlich gemacht, daß eine "geometrischebasierte" Prototyptheorie, die Begriffe durch Quadrupel $<D, d, p, c>$ (vgl. Abschnitt 1.2) konstituiert, inhaltlich inadäquat ist (s. 2.B. Osherson/Smith 1981, 1982; Tversky/Kahnemann 1983; Kamp/Partee 1989). Ähnlich wie das Problem der Inferenz erscheint mir der Aspekt der Kombinatorik besonders geeignet, Randbedingungen für die Theorienbildung zu setzen, die über die Auflistung einzelner, zu erklärender Fakten hinausgeht. $\mathrm{DaB}$ die damit verbundenen Herausforderungen an eine zu schaffende Prototyptheorie im Rahmen einer modelltheoretischen Semantik wohl am ehesten einzulösen sind, dafür gibt der genannte Aufsatz von Kamp/Partee zahlreiche Hinweise. Dennoch ist einzugestehen, daß wir noch weit entfernt von der Formulierung einer wirklichen, e i n h e i t l i ch e $n$ Prototyptheorie stehen - einer Theorie, welche die Phänomene der Inferenz ebenso behandelt wie die Phänomene der Kombinatorik und dabei auch nicht den Bereich ausklammert, der mit den Mechanismen der polysemen und metonymischen Interpretation zusammenhängt.

\section{Literatur}

Armstrong, S. L./Gleitman, L./Gleitman, H. (1983): What some concepts might not be. In: Cognition 13, S. 263-308.

Asher, N./Morreau, M. (1991): Commonsense entailment: A modal theory of nonmonotonic reasoning. In: van Eijck, J. (Hg.): Logics in AI - Proceedings of the European workshop JELIA 90 . Berlin.

Ballweg, J. (1994): Allgemeingültige Sätze - eine Herausforderung für die Prototypensemantik. (In diesem Band)

Bealer, G. (1982): Quality and concepts. Oxford. 
Bealer, G./Mönnich, U. (1989): Property theories. In: Gabbey, D./Guenthner, F. (Hg.): Handbook of philosophical logic. Vol. IV. Dordrecht.

Berlin, B./Kay, P. (1969): Basic color terms: their universality and evolution. Berkeley.

Bierwisch, M. (1989): Semantische und konzeptuelle Repräsentation lexikalischer Einheiten. In: Studia Grammatica XXII, S. 61-99.

Blutner, R. (1985): Prototyp-Theorien und strukturelle Prinzipien der mentalen Kategorisierung. In: Linguistische Studien (Reihe A) 125, S. 86-135.

Blutner, R. (1993): Logik von Standard-Annahmen. Unveröffentlichtes Manuskript. Max Planck Arbeitsgruppe Strukturelle Grammatik an der HumboldtUniversität Berlin.

Brewka, G. (1991): Nonmonotonic reasoning: logical foundations of commonsense. Cambridge.

Bruner, J. S./Goodnow, J. J./Austin, G. A. (1956): A study of thinking. New York/London.

Caramazza, A./Grober, E. (1977): Polysemy and the structure of the subjective lexicon. In: Rameh, C. (Hg.): Georgetown University roundtable on language and linguistics. Washington.

Carnap, R. (1947): Meaning and necessity. Chicago.

Chierchia, G./Turner, R. (1988): Semantics and property theory. In: Linguistics and Philosophy 11, S. 261-302.

Coleman, L./Kay, P. (1981): Prototyp semantics: the English verb lie. In: Language 57, S. 26-44.

Deane, P. D. (1988): Polysemy and cognition. In: Lingua 75, S. 325-361.

Erdmann, K. O. (1901): Die Bedeutung des Wortes. Leipzig.

Fillmore, C. (1975): An alternative to checklist theories of meaning. In: Proceedings of the 1st annual meating. Berkeley Linguistic Society, S. 123-131.

Geeraerts, D. (1987): On necessary and sufficient conditions. In: Journal of Semantics 5, S. 275-291.

Gougen, J. A. (1969): The logic of inexact concepts. In: Synthese 19, S. 325373.

Hobbs, J./Stickel, M./Martin, P./Douglas, E. (1988): Interpretation as abduction. In: Proceedings of the 26th Annual Meeting of the Association for Computational Linguistics. Buffalo, NY. S. 95-103.

Horty, F. J./Thomason, R. H./Touretzky, D. S. (1987): A clash of intuitions: The current state of nonmonotonic multiple inheritance systems. In: Proceedings of the 10th IJCAI, Milan, Italy.

Hunt, E. B. (1962): Concept learning: An information processing approach. New York/London.

Jackendoff, R. (1987): Consciousness and the computational mind. Cambridge, Mass.

Kamp, H./Partee, B. (1989): Prototype theory and compositionality. Unveröffentlichtes Manuskript. Universitāt Stuttgart. 
Katz, J. J. (1972): Semantic theory. New York.

Katz, J. J./Fodor, J.A. (1963): The structure of semantic theory. In: Language 39, S. 170- 210.

Keil, F. C. (1987): Conceptual development and category structure. In: Neisser, U. (Hg.): Concepts, and conceptual development. Cambridge etc.

Kripke, S. (1972): Naming and necessity. In: Davidson, D./Harman, G. (Hg.): Semantics of natural language. Dordrecht.

Kutschera, F. von (1975): Sprachphilosophie. 2. völlig neu bearbeitete und erweiterte Auflage. München.

Lakoff, G. (1987): Women, fire, and dangerous things: What categories reveal about the mind. Chicago.

Labov, W. (1973): The boundaries of words and their meanings. In: Bailey, C.-J. N./Shuy, R. W. (Hg.): New ways of analyzing variation in English, Vol. 1. Washington.

Langacker, R. W. (1986): Foundations of cognitive grammar. Vol. 1. Stanford.

Lehrer, A. (1970): Static and dynamic elements in semantics: hot, warm, cool, cold. In: Papers in Linguistics 3, S. 49-74.

Lehrer, A. (1978): Structures of the lexicon and transfer of meaning. In: Lingua 45, S. 95-123.

Lieb, H.-H. (1981): Wortbedeutung: Argumente für eine psychologische Konzeption. In: Lingua 52, S. 1-32.

Lutzeier, P. R. (1981): Wort und Feld. Wortsemantische Fragestellungen mit besonderer Berücksichtigung des Wortfeldbegriffs. Tübingen.

Marr, D. (1982): Vision. San Francisco.

Miller, G. A./Johnson-Laird, P. N. (1976): Language and perception. Cambridge, Mass.

Montague, R. (1974): Formal Philosophy, ed. Richmond Thomason. New Haven.

Nunberg, G. (1979): The non-uniqueness of semantic solutions: Polysiny. In: Linguistics and Philosophy 3, S. 143-184.

Osherson, D. N./Smith, E. E. (1981): On the adequacy of prototype theory as a theory of concepts. In: Cognition 9, S. 35-58.

Osherson, D. N./Smith, E. E. (1982): Gradedness and conceptual combination. In: Cognition 12, S. 299-318.

Pulman, S. G. (1983) Word meaning and belief. London.

Putnam, H. (1975): The meaning of "meaning”. In: Gunderson K. (Hg.): Language, mind, and reality, Minnesotoa studies in the philosophy of science. Vol. 7. Minneapolis.

Rosch, E. (1975): Cognitive reference points. In: Cognitive Psychology 7, S. 532-547.

Rosch, E. (1977): Human categorization. In: Warren, W. (Hg.): Studies in cross-cultural psychology. Vol. 1. New York. 
Rosch, E. (1978): Principles of caregorization. In: Rosch, E./Lloyd, L.L. (Hg.): Cognition and categorization. Hillsdale, N.J.

Rosch, E./Mervis, C. B. (1975): Family resemblances: Studies in the internal structure of categories. In: Cognitive Psychology 7, S. 573-605.

Rosch, E./Mervis, C. B./Ciray, W. D./Johnson, M./Boyes-Bream (1976): Basic objects in natural categories. In: Cognitive Psychology 8, S. 382-439.

Schwartz, S. P. (1979): Natural kind terms. In: Cognition 7, S. 301-315.

Searle, J. R. (1980): The background of meaning. In: Searle, J. R./Kiefer, F./Bierwisch, M. (Hg.): Speech act theory and pragmatics. Dordrecht. S. 221232.

Shepard, R. N./Hovland, C. I./Jenkins, H. (1961): Learning, and memorization of classifications. In: Psychological Monography 75, 13 (ganzes Heft).

Smith, E./Medin, D. (1981): Categories and concepts. Cambridge, Mass.

Sweetser, E. E. (1984): Semantic structure and semantic change. Ph. D. Dissertation, University of California, Berkeley.

Tsohatzidis, S. L. (1990): A few untruths about lie. In: Tsohatzidis, S. L. (Hg.): Meanings and prototpyes. London/New York.

Tversky, A./Kahnemann, D. (1983): Extensional versus intuitive reasoning: The conjunction fallacy in probability judgment. In: Psychological Review 90, S. 293-315.

Veltman, F. (1991): Defaults in update semantics. In: ITLI prepublications for logic, semantics and philosophy of language, University of Amsterdam.

Waismann, F. (1952): Verifiability. In: Flew, A. (Hg.): Logic and Language. Oxford. S. 117-144.

Weinreich, U. (1966): Explorations in semantic theory. In: Sebeok, T.A. (Hg.): Current trends in linguistics. Vol. 3. The Hague. 